\title{
Dediche di armi nei santuari sannitici*
}

\author{
Gianluca Tagliamonte \\ Universitá degli Studi di Lecce
}

\section{Riassunto}

Sulla base dell'esame della documentazione archeologica disponibile proveniente da santuari e luoghi di culto del Sannio, questo contributo cerca di analizzare alcuni aspetti connessi all'uso della dedica di armi nel mondo sannitico e di evidenziare la complessità di tale fenomeno. Per quanto riguarda il principale luogo di culto sannita (il noto santuario pentro di Pietrabbondante), la notevole presenza di armi e parti dell'armamento fra i materiali votivi ne rivela chiaramente il ruolo centrale nelle forme ideologiche di auto-affermazione e auto-rappresentazione sannitiche. Sembra plausibile interpretare le armi e le parti dell'armamento dedicate sia come preda di guerra (skyla o làphyra apò tòn polemìn) che come armi proprie del dedicante (òpla oìs autòs echreito). Per quanto concerne altri santuari e luoghi di culto sannitici (Campochiaro, Schiavi d'Abruzzo, Valle d'Ansanto, ecc.), pare verosimile ritenere che la presenza di armi e parti dell'armamento dedicate sia riconducile a offerte individuali, connesse a dediche private (òpla oìs autòs echreito), pratiche iniziatiche, riti di passaggio, ecc.

\begin{abstract}
Based on the analysis of the available archaeological evidence coming from Samnite sanctuaries and cult places, this paper aims at stressing some aspects related to the use of the dedication of weapons among the Samnites and at demonstrating the complexity of such a phenomenon. With respect to the main Samnite cult place (the well-known Pentrian sanctuary of Pietrabbondante), the considerable presence of arms and armour among the votive offerings clearly shows its central role in Samnite ideological forms of self-assertion and self-representation. It seems plausible to interpret dedicated arms and armour both as spoils of war (skyla o làphyra apò tòn polemion) and as the dedicator's own weapons (òpla oìs autòs echreìto). With reference to other Samnite sanctuaries and cult places (Campochiaro, Schiavi d'Abruzzo, Valle d'Ansanto, ecc.), it seems likely that the presence of dedicated arms and armour can be due to individual offerings, related to private dedications (òpla ois autòs echreito), initiation practices, transition rites, ecc.
\end{abstract}

Keywords: Samnite sanctuaries, warfare, religion, dedication of arms and armour.

Nel corso degli ultimi anni, anche e soprattutto a seguito di scoperte archeologiche talora davvero notevoli ${ }^{1}$, il tema della dedica delle armi nei

* Ringrazio gli organizzatori del Seminario, Proff. Manuel Bendala, Pierre Moret, Fernando Quesada Sanz, per il gentile invito rivoltomi a partecipare all'incontro madrileno e per la cortese ospitalità nella bella Casa de Velázquez. Il mio ringraziamento si estende agli amici e colleghi Katia Mannino ed Eugenio Polito, ai quali devo consigli e suggerimenti, e ad Alessandra Villone, funzionario responsabile dell'Archivio fotografico della Soprintendenza per i Beni Archeologici santuari italici e italioti del Meridione d'Italia, nonché in quelli della Sicilia greca e indigena, ha in più occasioni sollecitato l'interesse degli stu-

delle Province di Napoli e Caserta, e Gianni Ruggiero, tecnico del Laboratorio fotografico del Dipartimento di Beni Culturali dell'Università di Lecce, per l'aiuto fornitomi nel reperimento e nella riproduzione fotografica delle immagini poste a corredo di questo testo.

1 Come, ad esempio, nel recente caso del santuario in località Imbelli di Campora San Giovanni, nel territorio dell'antica Temesa, contesto di cui è stata fornita una pronta edizione ad opera di La Torre, 2002. 
diosi italiani. Per limitarsi all'ultimo decennio, non sono pochi al riguardo, infatti, i contributi già apparsi o dei quali è attesa l'edizione ${ }^{2}$. Né diversamente stanno le cose, del resto, se si guarda al mondo greco della madrepatria e ai numerosi e importanti lavori che sull'argomento sono stati prodotti o comunque editi in tempi recenti o recentissimi: si pensi, ad esempio, agli ultimi studi relativi alle armi dedicate nel santuario di Olimpia $^{3}$, o al volume di A. Jacquemin sul rapporto fra guerra e religione ${ }^{4}$, se non ai diversi articoli apparsi nel corso degli anni Novanta ${ }^{5}$.

In questi e in altri studi, che si sono aggiunti a una bibliografia specifica già abbastanza cospicua e nella quale spiccavano, sul versante greco, i nomi di R. Lonis ${ }^{6}$ e W.K. Pritchett ${ }^{7}$, il tema della presenza di armi nei contesti santuariali del mondo greco (della madrepatria e delle colonie d'Occidente) è stato esaminato da una molteplicità di punti di vista, che hanno privilegiato ora l'una ora l'altra delle fonti disponibili (archeologiche, epigrafiche e letterarie) e che hanno contemplato approcci di genere molto diverso: da quelli, prettamente archeologici, di tipo descrittivo o classificatorio-tipologico ${ }^{8}$, ad altri di carattere storico-religioso, storico-sociale, sociologico, ecc. ${ }^{9}$ Nel complesso tali studi, attraverso un esame delle forme ideologiche e materiali assunte dal fenomeno in questione, ne hanno evidenziato la complessità e la valenza polisemica: aspetti questi non riassumibili e risolvibili unicamente nel significato principale che senza dubbio esso viene ad avere, ovvero quello rappresentato dalla pratica, tutta bellica, della dedica delle armi sottratte al nemico vinto o morto (skỳla o làphyra apò tòn polemion). A fronte del valore paradigma-

\footnotetext{
2 Originati prevalentemente da interessi ed esigenze connesse allo studio e all'edizione di complessi archeologici indagati in epoche a noi più o meno vicine, tali studi hanno riguardato il mondo siceliota (Lentini, 2000; Spatafora, 2000; Di Vita, 2003; Spatafora, c.s.) e italiota (Parra, 1996; Sabbione, 1996 a; 1996 b; 1996 c; Rouveret, 2000; Cardosa, 2002; Cardosa, c.s.; Lombardo, c.s.; Parra, c.s.), nonché l'ambiente indigeno dell'Italia meridionale (Mazzei, 1996, 121; Masseria, 1999; Osanna, 2001; La Torre, 2002; La Torre, c.s.).

3 Ad es., Kunze, 1991; 1994; Baitinger, 2001; Philipp, 2004.

4 Jacquemin, 2000.

5 Ad es., Jackson, 1991; Philipp, 1991; Moustaka, 1994; Philipp, 1994; Jacquemin, 1999; Jost, 1999 a; 1999 b; Jacquemin, 2000: 162 ss.; Martelli, 2003.

6 Lonis, 1979.

7 Pritchett, 1979.

8 Cfr. i lavori citt. (supra nt. 3), rivolti soprattutto a una
}

tico e normativo che questa ultima in certi casi aveva finito con l'assumere, l'analisi del dato contestuale ha portato a formulare interpretazioni diverse, che evocano riti di passaggio e scenari di tipo iniziatico ${ }^{10}$; richiamano particolari esigenze e valenze di ordine rituale ${ }^{11}$; rinviano al rapporto intercorrente, sul piano funzionale e simbolico, in termini connotativi, fra divinità destinataria dell'offerta e l'offerta stessa ${ }^{12}$; sottolineano il ricorso alla pratica della dedica delle armi proprie (òpla ois autòs echreito) da parte del guerriero $^{13}$. Ciò, ferma restando la valenza prevalentemente gratulatoria della dedica di armi (e, dunque, la loro natura di veri e propri ex voto $)^{14}$, un aspetto questo la cui percezione si chiarisce essenzialmente grazie al contributo della documentazione letteraria ed epigrafica.

Rispetto al mondo greco (ivi compreso quello italiota e siceliota), per quello italico del Meridione della Penisola disponiamo di una base documentaria certo meno ampia e articolata. Se si eccettuano infatti alcuni sporadici ma importanti casi di iscrizioni italiche incise su elmi presumibilmente dedicati nel corso del IV sec. a.C. in santuari di area magno-greca ${ }^{15}$, le nostre conoscenze si basano in modo pressoché esclusivo sulla documentazione archeologica, pur potendosi ipotizzare, almeno in qualche circostanza, eventuali collegamenti a iscrizioni di dedica, il cui oggetto non sia esplicitato ${ }^{16}$. In seno a tale documentazione, quella relativa al mondo sannitico sembra avere, come noto, un peso particolare. Ed è di essa che in questa sede ci si vuole più direttamente occupare.

A tale riguardo occorre fare una precisazione preliminare, anche al fine di evitare possibili

classificazione tipologica dei materiali elaborata su base morfologico-formale.

9 Cfr. supra nt. 5.

10 Ad es., per il mondo greco: Moustaka, 1994: 36; Martelli, 2003: 469. Per quello italiota e italico-meridionale: Masseria, 1999: 489-490; Osanna, 2001: 65, 67; Cardosa, 2002: 100, 102.

11 Ad es., Lentini, 2000: 158-159; Parra, c.s.

12 Ad es., Philipp, 1992: 40; Sabbione, 1996 c: 156; Jost, 1999 a: 163 ss.; 1999 b: 131.

13 Pritchett, 1979: 245 ss.; cfr. Tagliamonte, 1989-1990: 525; Sabbione, 1996 c: 156.

14 Considerazioni al riguardo in Lonis, 1979: 148 ss.; Jackson, 1991: 237 ss.; Jacquemin, 1999: 142; Jost, 1999 a: 172 ss.

15 Cfr. infra nt. 108.

16 Un caso di questo genere potrebbe essere proprio quello della laminetta bronzea da Pietrabbondante, con iscrizione osca di dedica alla Vittoria: infra nt. 165. 


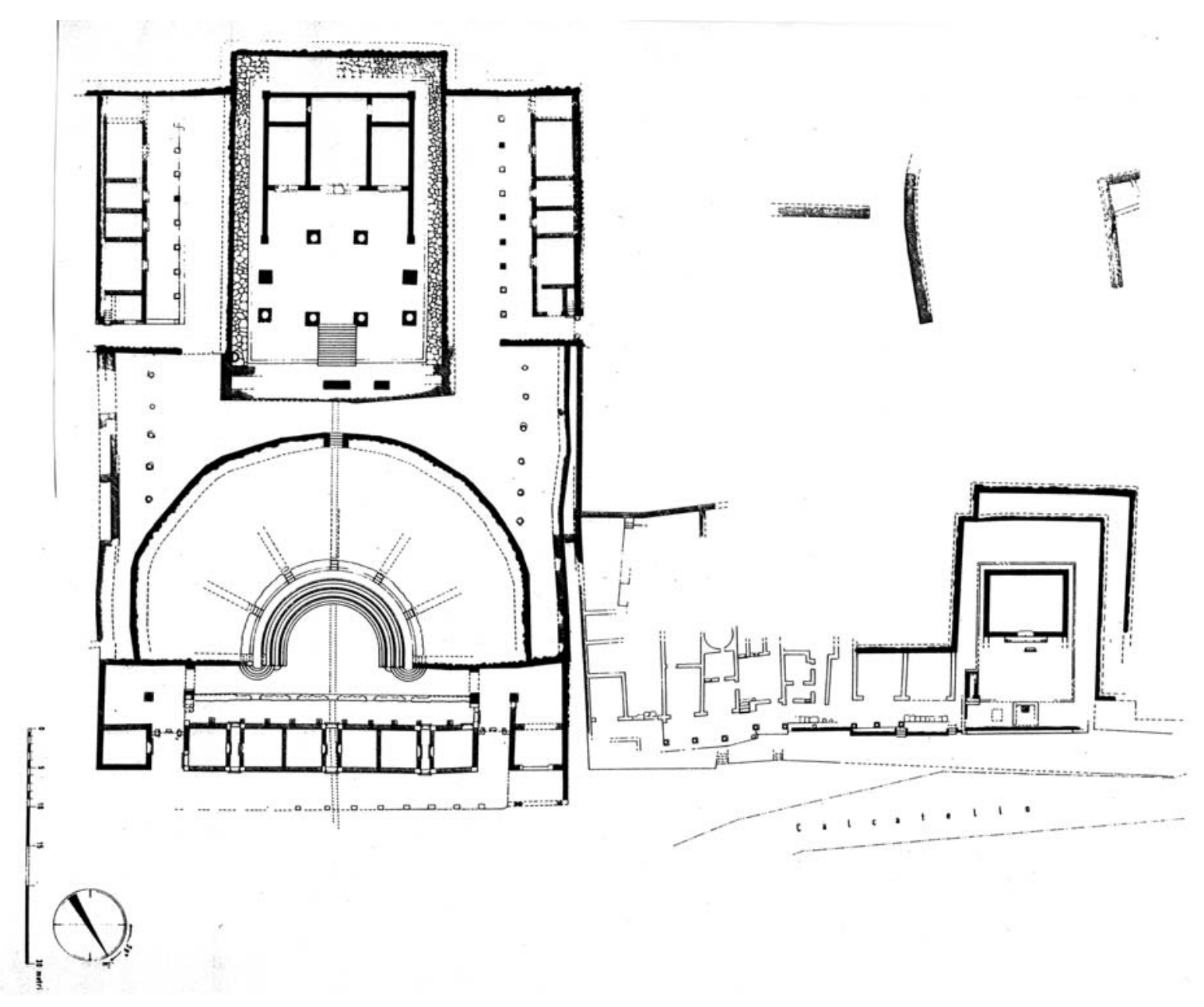

Fig. 1 - Pietrabbondante: planimetria del santuario (da La Regina, 1989: tav. I, i).

equivoci e fraintendimenti circa l'effettiva valenza che, sul piano etnico-territoriale, all'aggettivo sannitico viene attribuita: questo viene qui inteso nella sua accezione propria, riferito cioè ai territori nei quali in età storica erano stanziate le popolazioni (o, se si vuole, le tribù) di Carricini, Pentri, Frentani, Caudini e Irpini ${ }^{17}$.

All'interno del mondo sannitico così definito, una particolare rilevanza assume, ai fini del discorso che si intende qui affrontare, il caso di Pietrabbondante ${ }^{18}$, sede del principale - e più noto - santuario dei Sanniti Pentri, ubicato a circa 1000 m di altezza, in località Calcatello, su un

17 Dunque, nel senso indicato in Tagliamonte, 1997. Ne restano pertanto esclusi i Campani e le restanti genti di stirpe sannitica dell'Italia meridionale (Lucani e Brettii), che studi più o meno recenti (ad es., Saulnier, 1983; Schneider Hermann, 1996) continuano, troppo semplicisticamente, a etichettare come Sanniti, nonostante le sostanziali forme di differenziazione rilevabili, sul piano strutturale, nei rispettivi sistemi sociali e culturali. pendio digradante verso l'alta valle del Trigno (Fig. 1). Una tale rilevanza deriva dall'oggettiva, considerevole, consistenza che la presenza di armi e di parti dell'armamento, intere o frammentarie, assume in seno ai materiali votivi restituitici dagli scavi del monumentale e scenografico complesso santuariale, nonché dall'eco che tale presenza venne ad avere, nella letteratura archeologica italiana e non, sin dalla seconda metà dell'Ottocento, soprattutto dal momento in cui con le pubblicazioni di G. Fiorelli ${ }^{19}$ e M. Ruggiero ${ }^{20}$ se ne diede conto alla comunità degli studiosi ${ }^{21}$.

18 Rinvii alla ormai vasta bibliografia su Pietrabbondante in Storti, 1994, e in Capini, De Benedittis, 2000: 93-94.

19 Fiorelli, 1869: 7-10, n. 63 ss.

20 Ruggiero, 1888: 614 ss.

21 Proprio le scoperte di Pietrabbondante (con quelle, più o meno contemporanee, relative all'antica Capua) contribuirono in modo determinante all'affermazione di una nozione di "arte sannitica", o di "stile sannitico", 


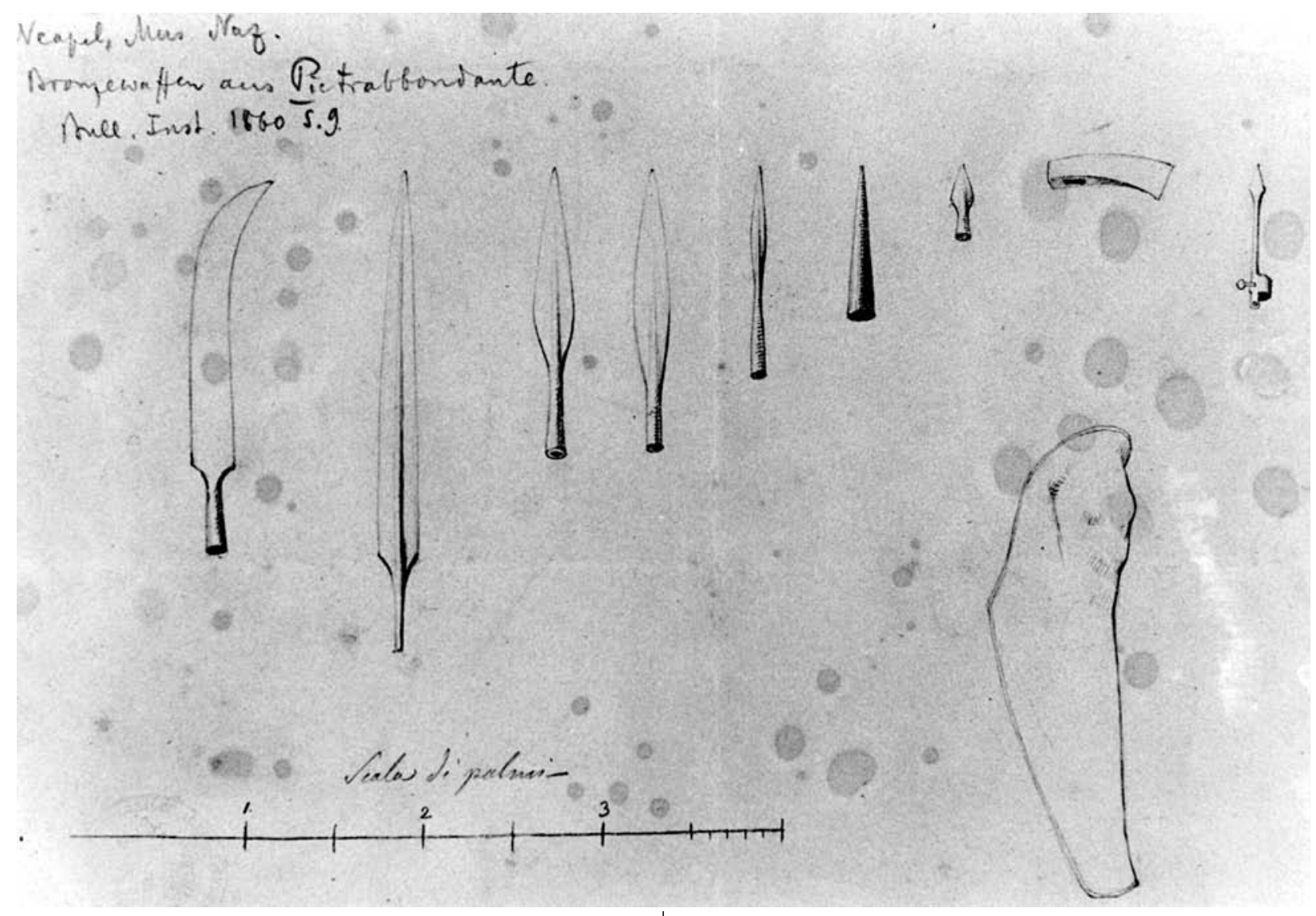

Fig. 2 - Armi dagli scavi borbonici di Pietrabbondante (Deutsches Archälogisches Institut Rom, Archivio disegni, cassetto 49, cartella P, 1860.36 g; cortesia T. Fröhlich).

Cospicue quantità di armi, offensive e difensive, e di altri oggetti ideologicamente e funzionalmente riferibili alla sfera della guerra, come noto $^{22}$, vennero portate alla luce già nel corso degli scavi intrapresi (ufficialmente, a partire dal 3 ottobre 1857) dai Borboni nell'area del santuario (Fig. 2) e confluirono, pertanto, nelle raccolte dell'attuale Museo Archeologico Nazionale di Napoli. A queste si aggiunsero, a oltre un secolo di distanza, quelle rinvenute in occasione delle nuove campagne di scavo archeologico condotte da A. La Regina, a partire dal 1959, nell'area del santuario $^{23}$.

e, più in generale, al riconoscimento di una specificità culturale e linguistica riferita ai Sanniti della tradizione storica: Minervini, 1858: 189; Garrucci, 1860: 8-9; De Petra, 1870: c. 119; Caraba, Cremonese, 1871: 9, 20; Caraba, 1873: c. 400.

22 Si vedano al proposito Fiorelli, 1869: 7-10; Ruggiero, 1888: 614 ss.; Isernia, 1980: 139-140, 143-145, 151-152.

23 Tali materiali, conservati nei depositi della Soprintendenza per i Beni Archeologici del Molise, sono in parte editi in Isernia, 1980: 140-142, 145-150, 151-153; Milano, 1991: 157-159.

24 Fiorelli, 1869: 7-9, nn. 63-135.
Il quadro delle attestazioni è ampio ed eterogeneo, comprendendo, come detto, armi tanto difensive quanto offensive, in bronzo e in ferro (Fig. 3). Già edite nel catalogo di Fiorelli (almeno per quanto riguarda gli esemplari pervenuti e custoditi nel Museo Archeologico Nazionale di Napoli) $)_{24}$ e poi puntualmente riproposte al pubblico e agli studiosi in occasione delle tre mostre italiane sui Sanniti svoltesi nel $1980^{25}, 1991^{26}$ e $2000^{27}$, tali armi includono infatti, stando almeno a quanto sinora edito, elmi del tipo suditalico-calcidese (Fig. 4) e "a bottone" (Fig. 5), nonché frammenti di altri elmi non classificabilii ${ }^{28}$; un

25 Isernia, 1980: 139-153.

26 Milano, 1991: 157-159.

27 Roma, 2000: 40-42. All'edizione romana della mostra hanno fatto seguito, con qualche variazione nella selezione dei materiali proposti, quelle di Santa Maria Capua Vetere e di Benevento (quest'ultima ancora aperta al pubblico, al momento, fine giugno 2004, di licenziare questo testo per la stampa).

28

Stando almeno a quanto riportato in Ruggiero, 1888: 631, per i ritrovamenti effettuati il 4 giugno 1858 (cfr. anche ibid.: 615, per un cenno ai resti di "un cimiero"). Interessante anche l'indicazione contenuta in Ruggiero, 


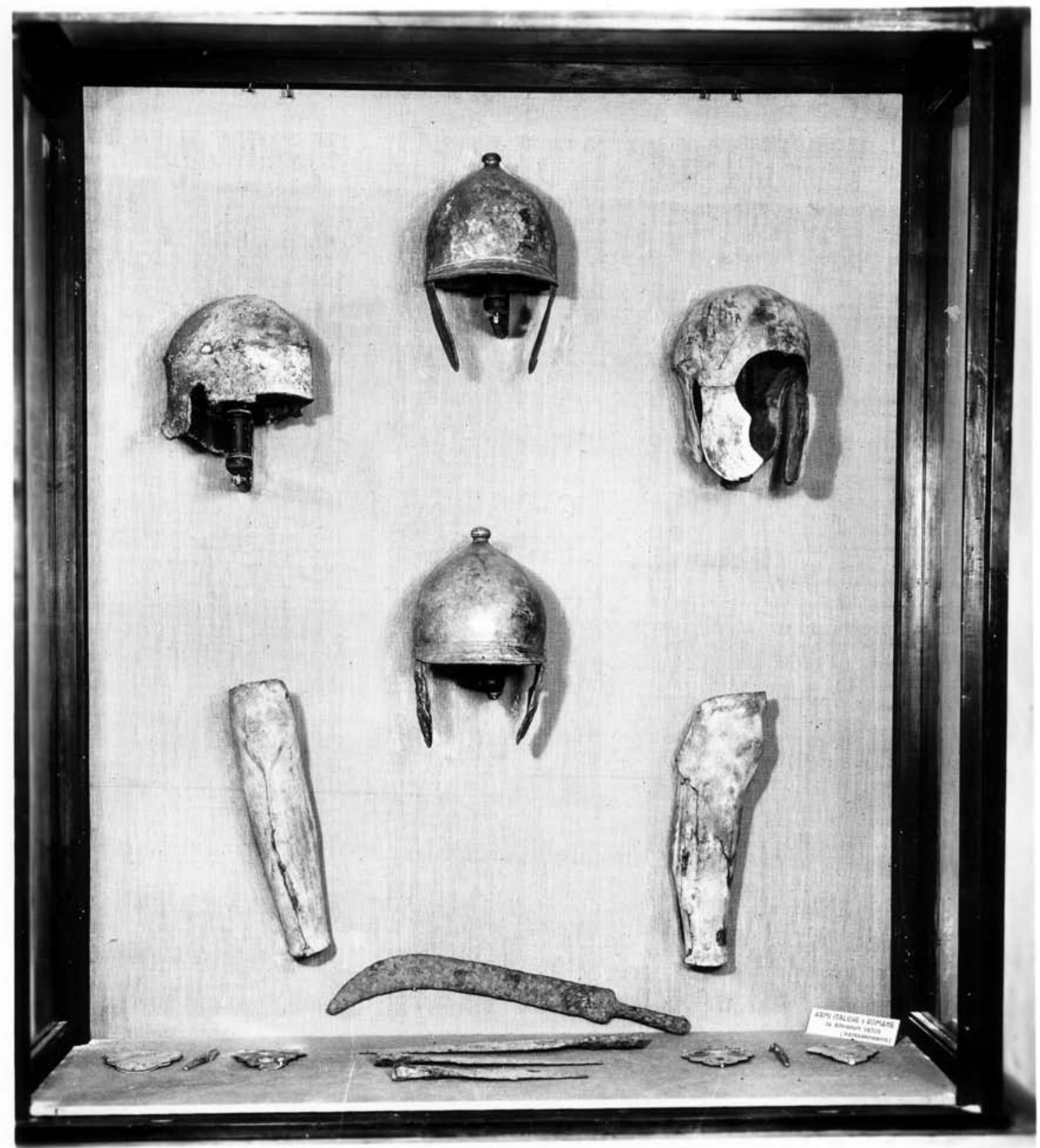

Fig. 3 - Museo Archeologico Nazionale di Napoli: vetrina con armi dal santuario di Pietrabbondante (vecchio allestimento) (Soprintendenza per i Beni Archeologici delle Province di Napoli e Caserta, neg. 2198).

gran numero di paragnatidi, decorate e non, del tipo anatomico o a tre dischi (Fig. 6), riferibili o presumibilmente riferibili ai due tipi di elmo sopra menzionati ${ }^{29}$; una singola, frammentaria ala (aletta) decorata di elmo, anch'esso verosimilmente di tipo suditalico-calcidese; schinieri anatomici; forse elementi di corazze; esemplari e

1888: 637 , che parrebbe riferibile a un altro frammento di paragnatide con decorazione figurata.

29 Più difficile pensare, nel caso delle paragnatidi bronzee a tre dischi, a un possibile riferimento a elmi con ganci di cinturoni di tipo "sannitico" (Fig. 7); spade lunghe, a doppio taglio, con relativi foderi in lamina di ferro; pugnali; una o più armi $(\mathrm{e} / \mathrm{o}$ strumenti) a lama ricurva; una scure; cuspidi di lancia e di giavellotto; saurotères; punte di freccia.

Se nel complesso le armi restituiteci dagli scavi borbonici vennero rinvenute nell'area

gola frontale di tipo B della classificazione di H. Pflug (1988 b: 291), che pure presentano questo genere di paraguance. 


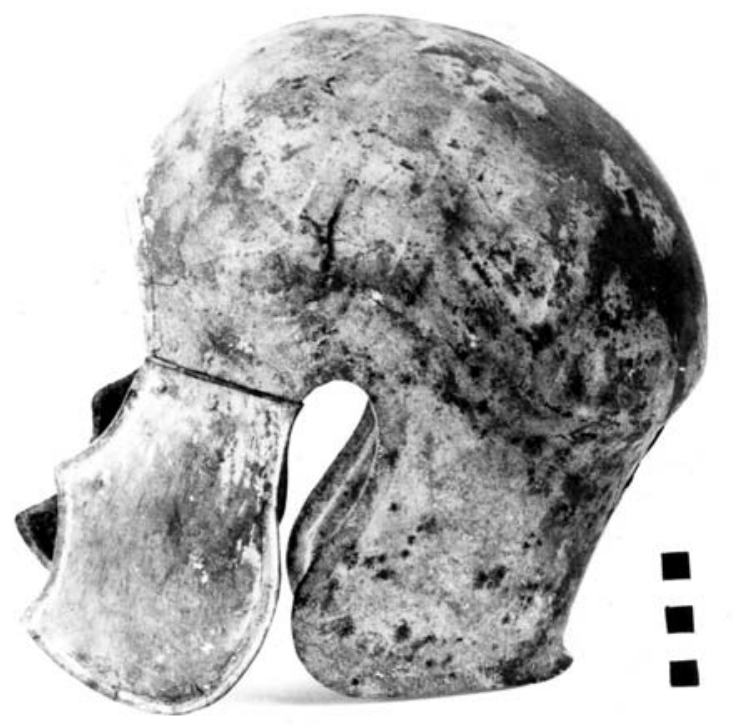

Fig. 4 - Museo Archeologico Nazionale di Napoli: elmo suditalico-calcidese con paragnatidi anatomiche dal santuario di Pietrabbondante (Soprintendenza per i Beni Archeologici delle Province di Napoli e Caserta, neg. n. 69219).

antistante il tempio $\mathrm{A}^{30}$, su una sorta di spianata obliterata da strati di abbandono e distruzione del santuario, quelle recuperate nelle indagini condotte in anni più recenti furono ritrovate per lo più in prossimità del tempio $\mathrm{B}^{31}$.

La registrazione di tale rilevante presenza, in una dimensione che a tutt'oggi resta con pochi confronti nell'Italia antica, ad opera degli scavatori ottocenteschi orientò la lettura archeologica che delle strutture rinvenute, o meglio di una parte di queste, essi proposero ${ }^{32}$, modificandone peraltro l'interpretazione data in un primo

30 Anche se numerosi furono i rinvenimenti di armi effettuati nel corso della prima campagna di scavo (svolta fra il 3 ottobre e il 28 novembre 1857), il nucleo più consistente di queste fu recuperato durante la successiva campagna di scavo, ripresa il 1 giugno 1858. In particolare, il ritrovamento di una gran quantità di armi è segnalato per il giorno 4 giugno e quelli seguenti, nel terzo "cavamento ..., ove si suppone potere esistere l'antico sepolcreto ... verso la china del monte Saraceno e dista dal teatro suddetto andando verso oriente pal. 250 circa” (Ruggiero, 1888: 631): dunque, grosso modo nell'area antistante il tempio oggi convenzionalmente definito A (un "palmo napolitano" corrispondeva a una misura di poco superiore ai $26 \mathrm{~cm}$ ).

31 Soprattutto negli spazi tra quest'ultimo e il teatro, nei livelli di interro e di scarico del tempio B, contenenti materiali della fase anteriore alla sua costruzione, nonché in quelli di riempimento delle concamerazioni

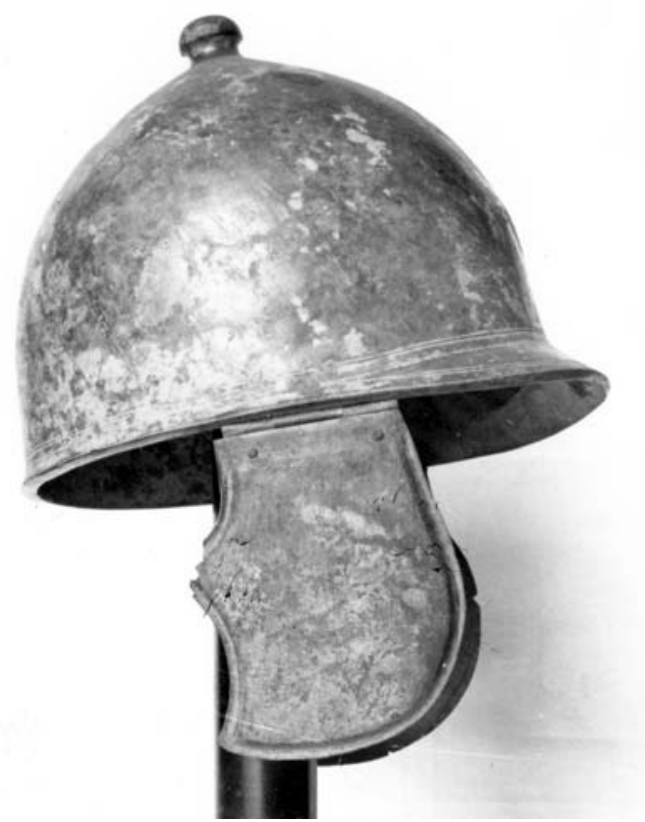

Fig. 5 - Museo Archeologico Nazionale di Napoli: elmo "a bottone" con paragnatidi anatomiche dal santuario di

Pietrabbondante (Soprintendenza per $i$ Beni Archeologici delle Province di Napoli e Caserta, neg. n. 22062).

tempo ${ }^{33}$. Il completamento delle indagini archeologiche e il ritrovamento di nuovi esemplari e frammenti di armi e armature dedicate a Calcatello fornirono poi, in tempi più recenti, ad A. La Regina l'opportunità per chiarire i termini del presumibile significato storico e funzionale che, nell'ambito di una più complessiva rilettura del santuario stesso, il fenomeno della dedica di armi venne lì ad assumere.

Una volta definito il preminente ruolo politico e religioso svolto dal complesso di Pietrabbondante in seno all'intero èthnos dei Sanniti

poste sotto il pavimento: Isernia, 1980: 142, 151.

32 Sulla base di tali rinvenimenti, le strutture messe in luce da U. Rizzi, Regio Architetto delle antichità di Capua e Pesto, e direttore della seconda campagna di scavo (1858) condotta a Pietrabbondante, vennero da questo identificate con un "tempio di Marte" (cfr. in tal senso la relazione di Rizzi ripresa da De Petra, 1870: c. 119, e da Ruggiero, 1888: 635). Successivamente, si vennero affermando le definizioni di "tempio sannitico" o "tempio italico": De Petra, 1870: c. 119; Caraba, Cremonese, 1871: 17; Caraba, 1873: c. 400; Maiuri, 1913: 456.

33 In precedenza in queste, infatti, si erano riconosciuti i resti di un "edificio pubblico" (Ruggiero, 1888: 616 ss., che si rifà alle osservazioni di G. Genovese, Architetto e Direttore dei Regi Scavi di Pompei, responsabile della prima campagna di scavo (1857) a Pietrabbondante), o di una "basilica" (Minervini, 1858: 187), o, ancora, di una "curia” (Mancini, 1899: 7, 34, 41 ss.). 


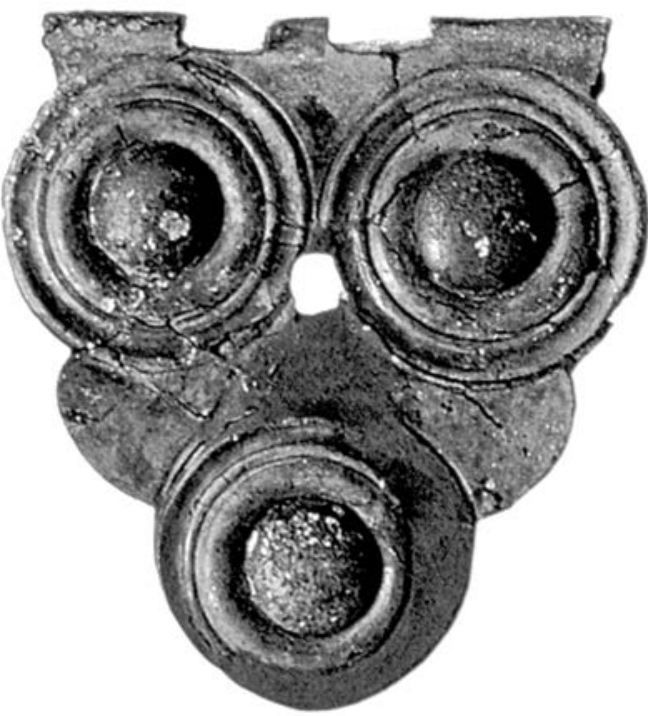

Fig. 6 - Soprintendenza per i Beni Archeologici del Molise, depositi: paragnatide a tre dischi di elmo dal santuario di Pietrabbondante (da Roma, 2000: 45 fig. 54).

Pentri ${ }^{34}$, tale significato veniva, in estrema sintesi, individuato nel valore di spolia hostium che le armi offerte nel santuario di Calcatello avrebbero assunto in quanto preda di guerra, acquisita in battaglia o nella spartizione del bottino: cioè, come armi sottratte al nemico sconfitto, vivo o morto $^{35}$. Le dediche collettive effettuate a nome di tutti i Pentri dai loro imperatores e duces fra la fine del V e la prima metà del III sec. a.C., all'indomani di vittorie riportate nel corso di spedizioni condotte in area magno-greca, delle cd. tre guerre sannitiche e degli avvenimenti immediatamente successivi, avrebbero, inoltre, dato

34 La Regina, 1970: 196; Strazzulla, 1973: 34-35; La Regina, 1976: 233; 1980: 131; 1984, 21-22; 1989: 303304, 422; 1990: 44; Capini, 1991: 113-114; Capini, De Benedittis, 19-20. Questo ruolo era stato percepito e inteso come "federale" da Lejeune, 1972.

35 In particolare, La Regina, 1984: 22-25; 1989: 303, 422.

36 La Regina, 1984: 24-25, sulla base delle note di scavo redatte da U. Rizzi e riprese da De Petra, 1870: c. 128, e da Ruggiero, 1888: 635. Rizzi registra, infatti, che il nucleo più consistente di armi (cfr. supra nt. 30) fu rinvenuto, a 4-5 palmi di profondità, nello scavo del "pubblico edificio" e che le armi furono trovate "alla rinfusa ed a cumuli e non appartenenti né ad avelli né ad altra distintiva posizione, ma su compatto e spianato suolo di terra che si succedeva dopo alcuni ciottoli di pietra mista con frr. di tegole e mattoni...”.

37 Oltre che da personali, specifici, interessi di studio, questa ricerca è stata originata anche da esigenze connesse alla redazione e alla realizzazione del progetto di luogo, per lo meno nel caso del nucleo di armi rinvenute negli scavi borbonici, all'erezione di un vero e proprio trofeo, in forma di congeries armorum, sulla spianata antistante il tempio $\mathrm{A}^{36}$.

Ora, se nel suo insieme la proposta formulata da La Regina sembra cogliere nel segno, una rilettura della documentazione nota ${ }^{37}$ offre tuttavia l'occasione per fornire qualche ulteriore elemento di valutazione e di precisazione in merito al tema della presenza delle armi nel santuario di Pietrabbondante $^{38}$. Essa consente, peraltro, di porre la questione in termini di maggiore problematicità e, verosimilmente, di attenuare la perentorietà delle affermazioni con le quali tale presenza è stata spiegata in esclusivo rapporto alla pratica della dedica delle armi in quanto preda di guerra $^{39}$.

Un primo ordine di considerazioni riguarda il gruppo di armi, o meglio di parti di armi, con decorazione figurata a sbalzo, restituiteci dal santuario e attribuite a produzione tarantina della fine del V e/o della prima metà del IV sec. a.C. ${ }^{40}$. In esso si è identificato il più antico nucleo di spolia hostium dedicati nell'area sacra e si è visto il riflesso di non meglio precisabili eventi bellici, localizzati o localizzabili in Magna Grecia in epoca antecedente le guerre sannitiche. Si tratta, come noto, di pezzi di grande pregio, integri o frammentari, tutti decorati a sbalzo, rinvenuti tanto negli scavi ottocenteschi quanto in quelli di epoca più recente: le due paragnatidi anatomiche, mobili, recanti figure di Nereidi (e delfino) in atto di consegnare (ad Achille) le armi (un elmo e una spada con elsa a crociera, in un caso; una coppia di schinieri anatomici, nell'altro), applicate all'elmo "a bottone" del Museo

allestimento scientifico di sette nuove sale della Sezione Topografica del Museo Archeologico Nazionale di Napoli, affidato a suo tempo allo scrivente dall'attuale Soprintendente Regionale per i Beni Culturali della Campania, Stefano de Caro. In una di queste sale, dedicate alle popolazioni preromane della Campania, saranno esposti anche i più significativi reperti da Pietrabbondante conservati nel museo napoletano.

38 Anche in relazione al quadro di attestazione di cui oggi, più in generale, si dispone per i luoghi di culto sannitici.

39 In particolare, La Regina, 1984: 22-25. Obiezioni e perplessità in merito alla univocità dell'interpretazione proposta da La Regina erano state, del resto, espresse già da D'Agostino, B., in Isernia, 1984: 44; Tagliamonte, 1989-1990: 529; Colonna, 1996: 46.

40 D'Agostino, 1980: 140-142; La Regina, 1984: 23-24; 1989: 303; Milano, 1991: 157-158. 

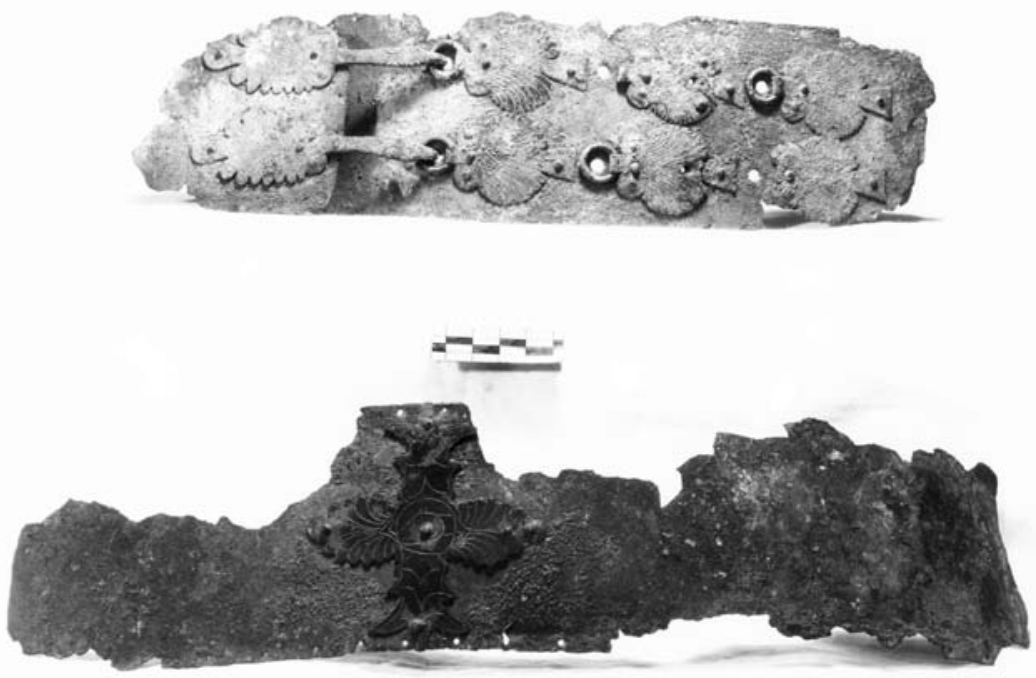

Fig. 7 - Museo Archeologico Nazionale di Napoli: cinturoni "sannitici" dal santuario di Pietrabbondante (Soprintendenza per $i$ Beni Archeologici delle Province di Napoli e Caserta, neg. 3128).

Archeologico Nazionale di Napoli ${ }^{41}$ (Figg. 8-9); le tre frammentarie lamine, pure riferibili o presu- mibilmente riferibili ad altrettante paragnatidi anatomiche mobili ${ }^{42}$, contraddistinte rispettiva-
41 Museo Archeologico Nazionale di Napoli, inv. n. 5744: Isernia, 1980: 143-145 n. 3, fig. 41.3, con rinvii alla bibliografia precedente. Belle riproduzioni fotografiche dell'elmo e delle paragnatidi in Roma, 2000: 40 fig. 45, 42 figg. 47-48.

42 La loro interpretazione funzionale come paragnatidi parrebbe sicura nel caso dell'esemplare contraddistinto dalla figura di Eracle (stante la presenza, sul margine superiore della lamina, dei resti di una cerniera); probabile o, quanto meno, possibile in quello degli altri due (sulla base della morfologia e delle dimensioni dei pezzi, nonché dei confronti istituibili: vedi infra). Per alcune lamine bronzee sbalzate avvicinabili a quelle di Pietrabbondante e riconducibili al gruppo che include i cd. "Siris bronzes" (elenco degli esemplari in Aitken, 1982), si è in passato avanzata l'ipotesi di riconoscervi elementi decorativi degli spallacci di corazze (Guzzo, 1993: 167-168, con rinvii bibliografici, ai quali aggiungi almeno Vermeule et al., 1988: 94 n. 119, relativo a un esemplare del Museum of Fine Arts di Boston). A tale riguardo si è, tuttavia, notato come corazze di questo tipo non risultino diffuse in Italia meridionale. Ora, se è vero che, come afferma Guzzo (1993: 167), "gli spallacci decorati delle raffigurazioni ceramografiche sembrano pertinenti più a corazze di tipo macedone che a quelle anatomiche documentate in uso" nel Meridione della Penisola, non mancano anche qui, però, per lo meno a livello iconografico, attestazioni della presenza delle prime (quelle a corsetto rigido con spallacci decorati): ne abbiamo testimonianza, ad es., per il IV sec. a.C., nella pittura funeraria apula, pestana e campana (ad es., Mannino, 2004:714 Pontrandolfo, Rouveret, 1992: 198 fig. 2, 199 fig. 4; Benassai, 2001: 184 fig. 195) e in quella vascolare italiota (ad es., Saulnier, 1983: 73, 76 figg. 1-3, 77 nn. 1-3; Schneider Hermann, 1996: pl.
79), in rapporto a contesti per i quali l'attendibilità del dato antiquario non viene generalmente posta in discussione (al di là di possibili forme di dipendenza iconografica da modelli esterni). Va inoltre ricordato, quantunque difficile appaia un suo riferimento alla problematica in questione, che, per lo meno nell'ambiente lucano del IV sec. a.C., è documentato l'uso militare di una falera (le ridotte dimensioni e la collocazione al centro del torace non consentono di definirlo kardiophylax) assicurata da corregge. Anche in questo caso ne abbiamo attestazione iconografica nella pittura funeraria (Pontrandolfo, Rouveret, 1992: 45, 46 fig. 37) e vascolare pestana e lucana (Saulnier, 1983: 73, 74 fig. 6, 75 n. 6; Schneider Hermann, 1996: pl. 69), ed, eccezionalmente, nella decorazione sbalzata della straordinaria corazza anatomica della tomba a camera di Marcellina di Santa Maria del Cedro (Laos): Guzzo, 1992: 25 ss. n. 4 (ma cfr. anche Bordenache Battaglia, 1979: tav. CXXXIX, su cista prenestina al British Museum di Londra). Un sistema di corregge e falera centrale del tutto simile sembra più tardi documentato, sempre per via iconografica, nell'armamento gladiatorio di avanzata età tardo-repubblicana, come testimonia, ad es., il noto rilievo dal Tevere conservato a Roma, nel Museo Nazionale Romano (Roma, 2001 a: 355 n. 68). Un altro ambito, che potrebbe forse essere in qualche rapporto con le lamine bronzee a decorazione figurata sbalzata non riferibili o non immediatamente riferibili a parti dell'armamento difensivo, è quello delle grandi bullae e dei pettorali metallici tardoclassici e protoellenistici, contraddistinti dal medesimo tipo di ornamentazione figurata; oltre che dal rinvenimento di qualche esemplare reale, ne abbiamo ancora una volta significative attestazioni a livello iconografico, come documenta, ad es., per il IV sec. a.C. (specie, 

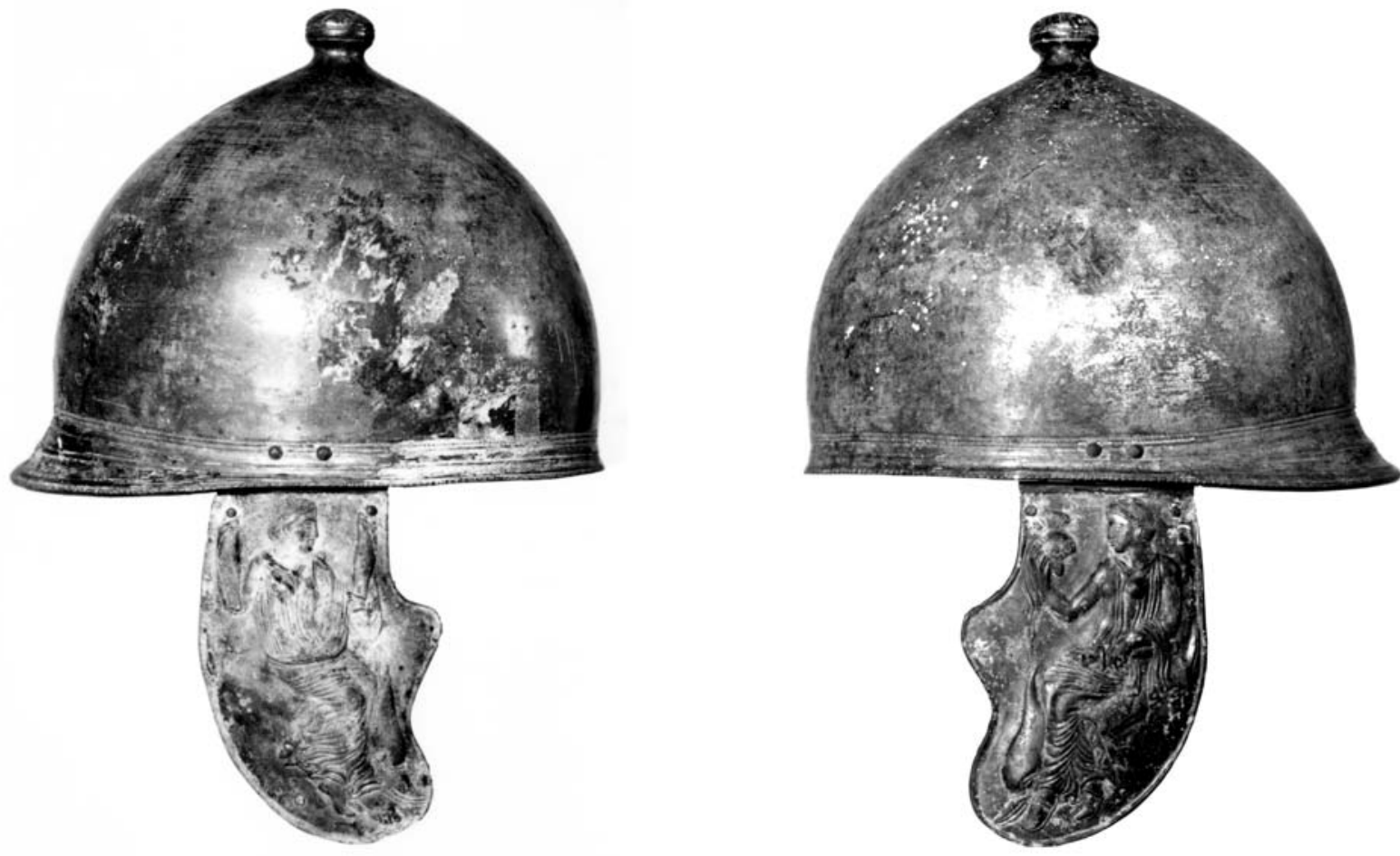

Figg. 8-9 - Museo Archeologico Nazionale di Napoli: elmo "a bottone" con paragnatidi anatomiche con decorazione figurata a sbalzo dal santuario di Pietrabbondante (Soprintendenza per i Beni Archeologici delle Province di Napoli e Caserta, neg. nn. 15062150638).

mente da raffigurazioni di Eracle in lotta con un avversario (Cicno?) ${ }^{43}$ (Fig. 10), di Amazzonomachia $^{44}$, di guerriero ritratto in nudità eroica ${ }^{45}$ (Fig. 11); l'ala (aletta sinistra) di elmo con rappresentazione di Nike tra elementi vegetali ${ }^{46}$ (Fig. 12); alcune paragnatidi anatomiche mobili decorate da ornati vegetali ${ }^{47}$ (Figg. 13-15).

per la seconda metà dello stesso) la serie di statue fittili di offerenti (maschili e femminili) rinvenute a Lavinium: Roma, 1981: 239-241 nn. D224, D225; 243 ss. nn. D227-D230; 254 ss. N. 241.

43 Soprintendenza per i Beni Archeologici del Molise, inv. n. 4437: Isernia, 1980: 149-150 n. 38, fig. 41.38; Milano, 1991: 158 n. d19; Roma, 2000: 47 fig. 57.

44 Soprintendenza per i Beni Archeologici del Molise, inv. n. 4435: Isernia, 1980: 148 fig. $41.36,150$ n. 36 (con rinvii alla bibliografia precedente).

45 Soprintendenza per i Beni Archeologici del Molise. Dell'esemplare, inedito, è stata resa nota una riproduzione fotografica in Roma, 2000: 44 fig. 50. Il pezzo ritrae, di profilo, un personaggio maschile seduto, o comunque addossato, a uno sperone roccioso. Luomo mostra folta chioma e lunga barba, e appare completamente nudo, ma con la clamide appoggiata sulla spalla destra e ricadente dietro la schiena. Nella mano destra stringe il fodero, nella sinistra la spada appena sguainata; dinanzi è uno scudo ovale. Desunta dal reperto-
Quantunque associate all'elmo "a bottone" di Napoli, è assai probabile che le due paragnatidi con figure di Nereide non siano ad esso pertinenti, come del resto già ipotizzato ${ }^{48}$, ma vadano piuttosto attribuite a un esemplare di tipo suditalico-calcidese, forse non dissimile da quello, con frontale, spirali temporali e paragnatidi anatomi-

rio iconografico greco, l'immagine potrebbe essere quella idealizzata di un guerriero oppure quella di un eroe del mito greco, Aiace, forse piuttosto che Achille o Eracle.

46 Soprintendenza per i Beni Archeologici del Molise, inv. n. 4436: Isernia, 1980: 148 fig. 41.37, 150 n. 37; Milano, 1991: 158-159 n. d21, 186 tav. 4d; Roma, 2000: 47 fig. 56.

47 Un primo esemplare (Soprintendenza per i Beni Archeologici del Molise, inv. 4433) è quello edito in Isernia, 1980: 147, 150 n. 34, 148 fig. 41.34 (con rinvii alla bibliografia precedente); Milano, 1991: 157-158 n. d17, 186 tav. 4d; Roma, 2000: 45 fig. 51. Un secondo (Soprintendenza per i Beni Archeologici del Molise, inv. 4434) è quello edito in Isernia, 1980: 150 n. 35 (con rinvii alla bibliografia precedente); Milano, 1991: 158 n. d18, 186 tav. 4d; Roma, 2000: 45 fig. 53. A questi se ne è aggiunto un terzo, di cui è fornita una riproduzione fotografica in Roma, 2000: 45 fig. 52.

48 Giampaola, 1980: 139. 


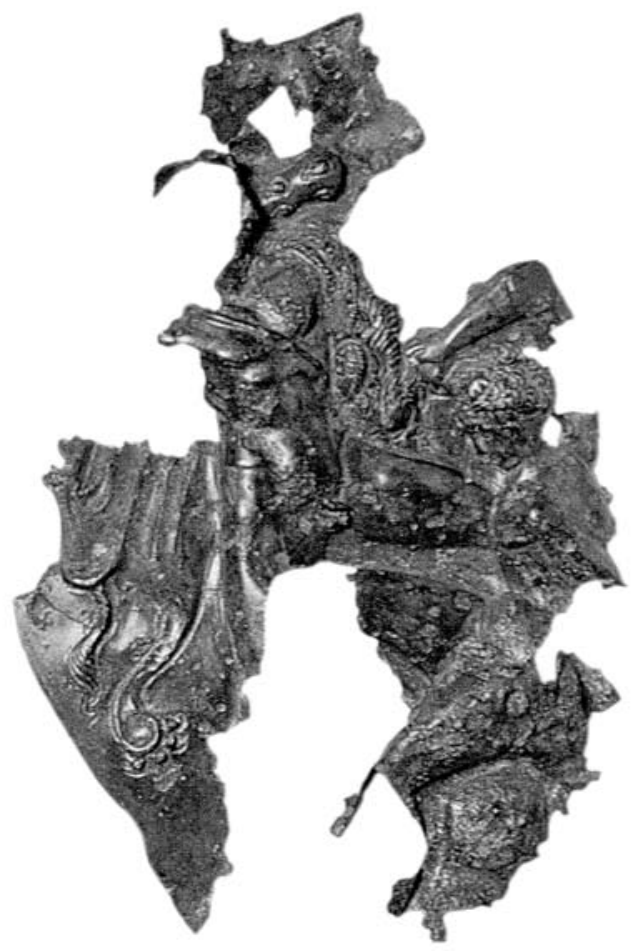

Fig. 10 - Soprintendenza per i Beni Archeologici del Molise, depositi: paragnatide di elmo con decorazione figurata a sbalzo dal santuario di Pietrabbondante (da Roma, 2000: 47 fig. 57).

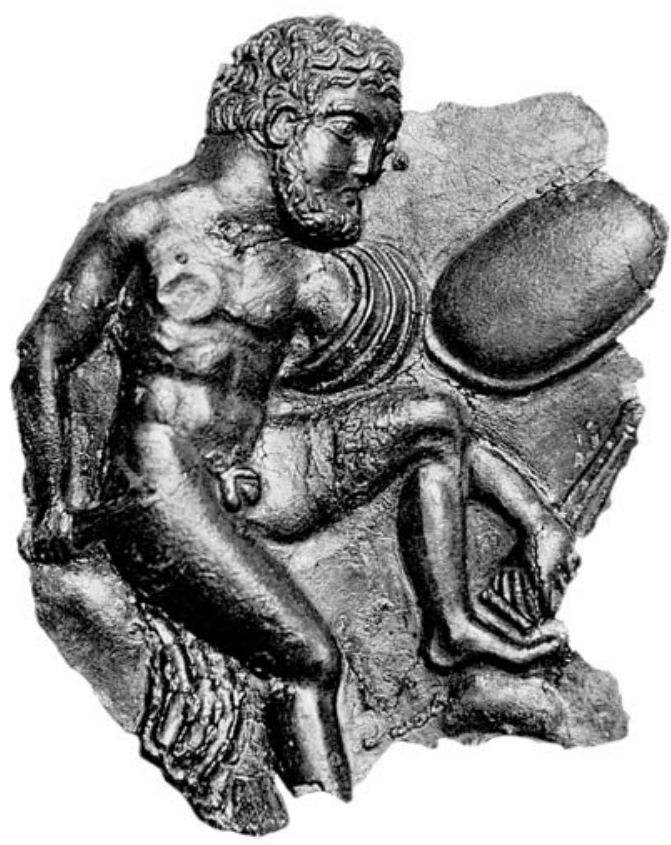

Fig. 11 - Soprintendenza per i Beni Archeologici del Molise, depositi: lamina con decorazione figurata a sbalzo dal santuario di Pietrabbondante (da Roma, 2000: 44 fig. 50). che a profilo anteriore bilobato, che proprio una delle Nereidi riprodotte sulle paragnatidi parrebbe stringere fra le mani ${ }^{49}$. A tale conclusione conduce la constatazione del fatto che tanto fra i "Realien" quanto nella documentazione iconografica disponibile nessun elmo "a bottone" presenta in genere le paragnatidi decorate ${ }^{50}$, risultando peraltro la decorazione figurata a sbalzo delle paragnatidi anatomiche mobili (ma con significative differenze) prerogativa di altri e in parte più antichi tipi di elmo in uso in Italia meridionale ${ }^{51}$. Inoltre, il profilo sinuoso, bilobato, del margine anteriore dei paraguance sembrerebbe maggiormente avvicinarsi più a quello che con-

49 La conformazione della calotta e la sua decorazione, nonché la resa, coerente e sufficientemente leggibile, dei dettagli farebbero escludere, per l'elmo riprodotto fra le mani della Nereide, altre possibilità di attribuzione, al di fuori di quella riferita alla classe suditalico-calcidese (sulla quale vedi infra nt. 51). Con una tale identificazione bene si accorda anche la presenza del cimiero dalla lunga "coda", che spesso vediamo contraddistinguere elmi di questa foggia, stando almeno alle testimonianze iconografiche (Saulnier, 1983: 60, 62 figg. 48, 63 nn. 4-8, 66-67; Schneider Hermann, 1996: 39-41, 44 , con rinvii alle relative illustrazioni). Del resto, ben diversa da quella attestata per l'esemplare da Pietrabbondante appare la resa di un elmo ascrivibile alla classe pseudo-attica su una paragnatide dei "Siris bronzes" al British Museum di Londra: da ultimo Bottini, 1992: 157 fig. 371; cfr. Dintsis, 1986, 129-130, taf. 54.2 .

50 Non può essere certo assimilata alle decorazioni che contraddistinguono le paragnatidi da Pietrabbondante (e le altre paragnatidi decorate, in genere: cfr. Aitken, 1982) quella (una piccola protome gorgonica) che è impressa sulle paragnatidi anatomiche mobili, a profilo anteriore bilobato, dell'elmo "a bottone" della tomba 669 di Lavello, loc. Cimitero, e che comunque costituisce un unicum (Bottini, Fresa, 1991: 58 n. 62*; Bottini, 1992: 156, 157 fig. 369). Stranamente, Guzzo (1993: 167) tende invece a ritenere preferenziale il rapporto fra paragnatidi decorate ed elmi "a bottone".

51 Nello specifico, elmi di tipo suditalico-calcidesi, "frigi" o "frigio-calcidesi" e pseudo-attici, solo per citare quelli che trovano effettiva, ancorché isolata, attestazione a livello di "Realien" in Italia meridionale. I primi sono stati in anni recenti così definiti (Pflug, 1988 a: 145 ss.; Cahn, 1989, 71; Bottini, 1991, 97-98; Bottini, A., in Melfi, 1993: 215-219; cfr. Kunze, 1994: 75 ss.) e appaiono ascrivibili al tipo 7 ("Helm mit Wangenklappen") della classificazione di Kunze (1967: 137; 1994: 69 ss.), corrispondente al tipo 5 di quella elaborata da Pflug (1988 a: 143 ss.); cfr. anche Dintsis, 1986: 138 ss. Fra di essi, si ricordino, ad es., gli esemplari di paragnatidi mobili decorate da protomi equine: Cahn, 1989: $93 \mathrm{n}$. 84 (di provenienza ignota); Bottini, 1991: 97 n. B3 (“da Capodignano” ?); Cahn, 1990: 114 n. 95 a (dalla Puglia); 
traddistingue le paragnatidi anatomiche associate ad elmi di tipo suditalico-calcidese che non a quello, più spigoloso, delle analoghe paragnatidi proprie degli elmi "a bottone" di tipo Coarelli $\mathrm{C}^{52}$. Del resto, come risulta dalla relazione di scavo ottocentesca ${ }^{53}$ e come già è stato osservato ${ }^{54}$, le due paragnatidi in questione non vennero trovate applicate all'elmo ma da questo separate, ancorché a breve distanza. Né va, infine, dimenticato che tutte le paragnatidi simili decorate, o presunte tali, sono state trovate sempre disgiunte dagli elmi cui verosimilmente dovevano essere riferibili ${ }^{55}$.

D'altronde, a elmi suditalico-calcidesi sembrerebbero in sostanza riconducibili anche le restanti paragnatidi decorate sopra menzionate, pur non senza dubbi dovuti al frammentario stato di conservazione delle stesse. Il tipo di decorazione figurata esibita sulle paragnatidi e il profilo delle lamine dalle quali sono costituite appaiono infat-

da protomi di ariete: Paestum 1996: 156 n. 61.6 (da Poseidonia, loc. Gaudo, tomba 164); Venezia, 1996: 650 n. 264.I (da Eboli, loc. Santa Croce, tomba 40); da protomi di grifo: Bottini, 1991: 97 n. A1 (“da Ruvo"); da protomi di aquila: Dintsis, 1986: taf. 66.3 (di ignota provenienza); ecc.; ulteriori esemplari sono segnalati in Aitken, 1982: 61. L'elmo suditalico-calcidese dalla tomba 1188 di Policoro, di recente edito (Roma, 2001 b: 91 n. 194), presenta paragnatidi mobili di forma circolare decorate da un motivo a ruota (molto vicino è l'esemplare presente sul mercato antiquario, ricordato da Kunze, 1994: 72 Abb. 70). Sugli elmi "frigi" o "frigiocalcidesi" vedi da ultimi, Adam, 1982: 7 ss.; Vokotopoulou, 1982: 497 ss.; Dintsis, 1986: 23 ss.; Waurick, 1988: 163 ss.; Pflug, 1989: 27-28; Guzzo 1990; 1992: 22-25. Accanto ad esemplari con paragnatidi con decorazioni che riproducono barba e baffi (ad es., Dintsis, 1986: taff. 10.5, 11.3, 11.4), ne abbiamo altri recanti paragnatidi decorate da raffigurazioni di sfingi (ad es., Dintsis, 1986: 218 n. K 53, taf. 19.4) o di grifi (Venezia, 1996: 653 n. 330 I). Sugli elmi pseudo-attici, da ultimi: Dintsis, 1986: 113 ss.; Waurick, 1988: 169 ss.; Pflug, 1989: 27-28. Per esemplari recanti paragnatidi con decorazioni figurate: ad es., Dintsis, 1986: 276-277 n. K 215, taf. 55.3.

52 Coarelli, 1976: 166-168, corrispondente al sottotipo Canosa della recente classificazione che degli elmi "a bottone", o, meglio, del tipo Montefortino (secondo la definizione adottata) ha fornito Junkelmann, 2000: 52 ss. Nonostante questo e altri studi apparsi nel corso degli ultimi anni (rinvii alla bibliografia in Tagliamonte, 2003 b: 159-160 nt. 106), la classe degli elmi "a bottone" attende ancora, per quanto riguarda l'area etruscoitalica, uno studio d'insieme.

53 Ruggiero, 1888: 631.

54 Giampaola, 1980: 139.

55 Come ancora osserva Giampaola, 1980: 139-140. Per ti difficilmente scindibili da un riferimento a quelle in genere associate agli elmi suditalico-calci$\operatorname{desi}^{56}$. E a un esemplare di questo tipo va poi, con tutta probabilità, attribuita anche l'aletta decorata con figura di Nike, che richiama morfologicamente e funzionalmente quelle, di rapace, applicate sui lati della calotta di elmi di questa classe ${ }^{57}$, piuttosto che quelle presenti su elmi "frigi" e "frigio-calcidesi" ${ }^{38}$.

Limmaginario evocato dalle rappresentazioni riprodotte sulle decorazioni figurate riflette un orizzonte ideologico omogeneo e compatto, saldamente improntato, nei suoi espliciti riferimenti, all'esaltazione dei valori della pratica bellica e dell'aretè guerriera. Per una - non saprei dire quanto - singolare coincidenza, le immagini in questione vengono a comporre, in qualche modo, le singole unità semantiche di un unico, coerente discorso narrativo e figurativo incentrato, come detto, sull'enfatizzazione dell'esperienza

un quadro complessivo delle attestazioni cfr. Aitken, 1982, integrato dalle osservazioni di Lippolis, 1984: 35, 40; Pflug, 1988 a: 145; Cahn, 1989: 24-25; Bottini, 1992: 156-158. Simile al caso di Pietrabbondante sembra quello di un paio di paragnatidi con raffigurazioni di guerrieri, provenienti dall'Italia meridionale e verosimilmente riferibili a un medesimo elmo (di tipo suditalico-calcidese?), di cui Aitken (1982: $61 \mathrm{nn}$. e, f) segnala la presenza sul mercato antiquario statunitense (ipotizzandone, però, l'originaria pertinenza a un contesto funerario). A differenza di quelle decorate con soggetti umani e/o divini, le paragnatidi ornate da protomi o figure animali sono state, in diverse circostanze, rinvenute insieme agli elmi suditalico-calcidesi cui esse erano riferibili (cfr. supra nt. 51).

56 Cfr. quanto osservato supra nt. 51.

57 A livello di "Realien", alette metalliche appaiono applicate agli esemplari elencati da Bottini, 1991: $97 \mathrm{nn}$. A2, B13, B14, 98 nn. B21-24, integrato, almeno, da Melfi, 1993: 215 ss. n. 31 (da Tricarico); Roma, 2001: 88 n. 155 (da Lavello, loc. Cimitero, tomba 686). Il riferimento dell'ala da Pietrabbondante a un elmo suditalico-calcidese era stato, del resto, già ipotizzato da S. Capini (Milano, 1991: 158-159 n. d21, 186 tav. 4d). Nella contemporanea documentazione iconografica non mancano esplicite attestazioni della presenza di alae su elmi suditalico-calcidesi: ad es., per la pittura funeraria pestana: Pontrandolfo, Rouveret, 1992: 281 fig. 3, 394-396 (Vannullo, tomba 2) e, probabilmente, 260 fig. 1, 380385 (Gaudo, tomba 2/1957); per quella campana: Benassai, 2001: 188 fig. 197 (Capua).

58 Come, ad es., negli esemplari da Conversano e Ruvo: Adam, 1982: 9 n. 3, 30 pl. IV e 10 n. 5, 28 pl. II b (per forma, dimensioni e resa dei dettagli, le alae qui presenti differiscono notevolmente da quelle applicate agli elmi suditalico-calcidesi). 


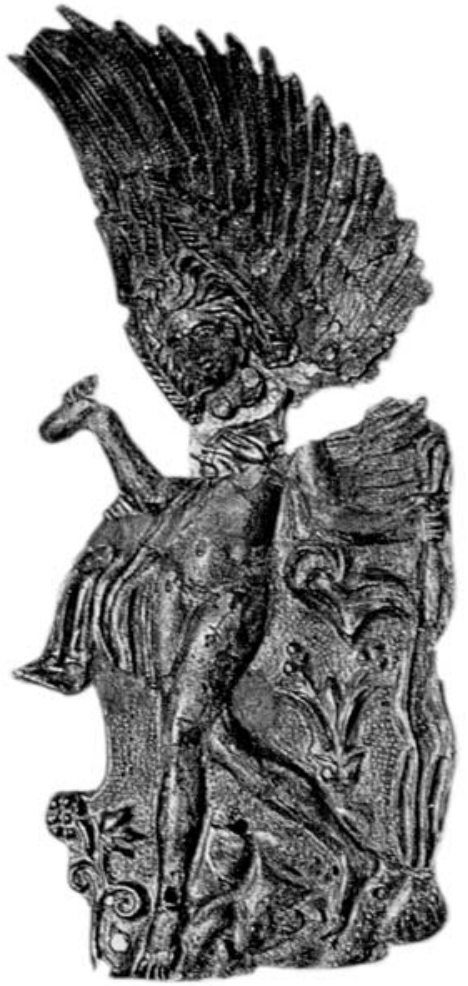

Fig. 12 - Soprintendenza per i Beni Archeologici del Molise, depositi: aletta di elmo con decorazione figurata a sbalzo dal santuario di Pietrabbondante (da Roma, 2000: 47 fig. 56).

guerriera. Di questa, tramite il ricorso e l'adozione di iconografie e di un linguaggio simbolico di matrice greca, sembrano illustrati e sintetizzati i momenti fondamentali: la preparazione per la guerra (richiamata, ovviamente, dall'episodio della consegna delle armi da parte delle Nereidi ad Achille); il combattimento, proiettato in una dimensione mitica ed eroica (Eracle, le Amazzoni), o quanto meno evocata come tale (il guerriero nudo, con spada sguainata) $)^{59}$; la celebrazione della vittoria sul nemico (Nike).

Si tratta di temi e iconografie che si affermano nel mondo italiota e, soprattutto, italico dell'Italia

59 A una tale interpretazione non osterebbe l'eventuale identificazione del personaggio con Aiace, potendosi supporre un riferimento a scene di combattimento, delle quali è protagonista l'eroe, o all'episodio della contesa per le armi di Achille (e, dunque, non necessariamente o direttamente a quello del suicidio dell'eroe): cfr. Touchefeu, O., in LIMC I.1, 1981, s.v. Aias I: 317 ss. n. 22 ss., 324 ss. n. 70 ss.

60 Considerazioni al proposito, ad es., in Guzzo, 1993: 168, 170-171; Mazzei, 1996: 122; Künzl, 1997: 73-74. All'immaginario della guerra nella produzione artistica e artigianale magno-greca dell'avanzato IV sec. a.C. è stata, inoltre, dedicata una sessione tematica (con inter- meridionale già a partire dalla seconda metà del $\mathrm{V}$ e che trovano la loro massima diffusione nel corso del IV sec. a.C. ${ }^{60}$. Il riferimento a modelli greci di autorappresentazione elitaria non appare disgiunto, e anzi ne è basilare elemento costitutivo, da quegli intenti di eroizzazione che a tale autorappresentazione palesemente ineriscono, come dimostra proprio il caso delle armi decorate di Pietrabbondante: le immagini trionfali ed eroiche che esse evocano non appartengono solo al mito ma anche alla storia personale del guerriero che tali armi possedeva, o per lo meno sono rivissute come tali. In rapporto all'affermazione e alla circolazione di questi modelli in ambito italico-meridionale nel periodo in questione va comunque segnalato il particolare valore documentario assunto dalle testimonianze di Pietrabbondante, risultando queste fra le non molte attestazioni di natura extra-funeraria di cui disponiamo al riguardo.

Quantunque già presenti in ambiente magnogreco nella seconda metà del V sec. a.C., è nel corso del IV secolo, come poc'anzi si accennava, che tali iconografie conoscono la loro massima diffusione nell'Italia meridionale. Ciò sembra valere anche per il tema riprodotto sui due esemplari meglio conservati della serie: le due paragnatidi applicate all'elmo del Museo Archeologico Nazionale di Napoli (Figg. 16-17). Il motivo della consegna delle armi ad Achille da parte delle Nereidi su esseri marini o in scene di ambientazione marina, già attestato su prodotti dell'artigianato artistico magno-greco, e tarentino in particolare, degli ultimi decenni del V secolo, si afferma pienamente nel corso del IV secolo (soprattutto dalla seconda metà dello stesso) ${ }^{61}$. E in effetti, a una datazione all'inoltrato IV sec. a.C. delle due paragnatidi, recenziore dunque rispetto a quella (inizi IV a.C.) già avanzata ${ }^{62}$, sembrerebbero condurre anche altri elementi. Se, sul piano stilistico, le annotazioni fatte ${ }^{63}$ non parrebbero fare escludere più tarde ipotesi di

venti di P. Orlandini, E. Mugione, K. Mannino) di Taranto, 2004. Osservazioni di carattere più generale nel recente contributo di Hölscher, 2003.

61 Si vedano al riguardo Besques, 1988: 10, 14 ss.: IcardGianolio, N., Szabados, A.-Y in LIMC VI,1, 1992, s.v. Nereides: 808-811, n. 315 ss.; Barringer, 1995: 39 ss., 178 ss. Ulteriori osservazioni ed esempi in Melfi, 1993: 225227 n. 33; De Filippis, 1995: 90 ss.; Romito, 1995: 33.

62 Giampaola, 1980: 139; cfr. La Regina, 1984: 23. Anche Bottini, 1992: 158 sembrerebbe datare le due paragnatidi a un momento precoce del IV sec. a.C.

63 Giampaola, 1980: 139. 

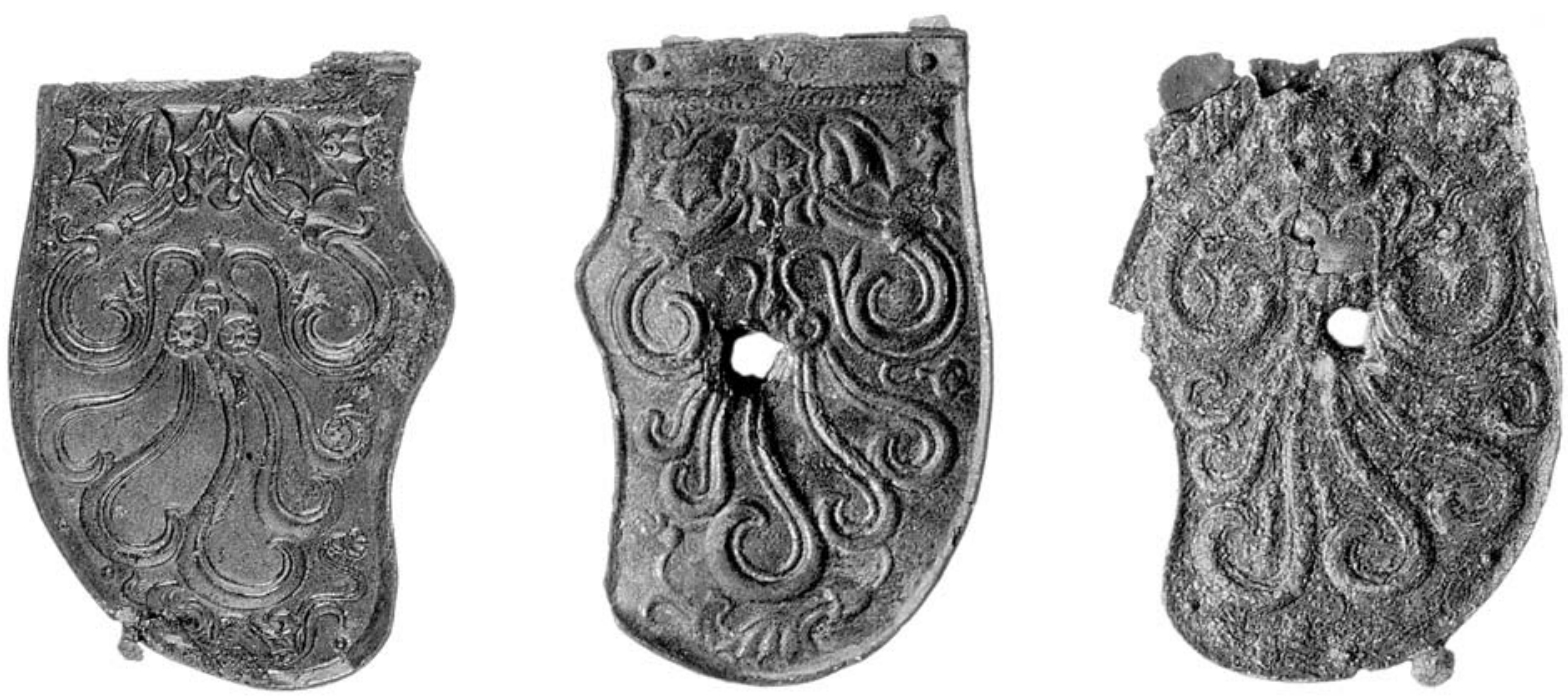

Figg. 13-15 - Soprintendenza per i Beni Archeologici del Molise, depositi: paragnatidi di elmo con decorazione figurata a sbalzo dal santuario di Pietrabbondante (da Roma, 2000: 45 fig. 51-53).

datazione, è soprattutto il dato antiquario a fornire qualche ulteriore indicazione in tal senso. L'elmo $^{64}$ che sorregge con la destra la Nereide raffigurata sulla paragnatide sinistra riproduce, come detto, un esemplare di tipo suditalico-calcidese, caratterizzato da decorazione a rilievo del frontale e da spirali temporali, nonché da paragnatidi anatomiche mobili bilobate: elementi che

64 Nella prospettiva antiquaria, indicazioni assai più generiche sono quelle desumibili dalle immagini della spada con elsa a croce e degli schinieri anatomici riprodotti fra le mani delle due Nereidi: armi delle quali è, ad ogni modo, testimoniato l'uso, per via archeologica, in contesti italici di IV sec. a.C.

65 Vedi quanto osservato supra nt. 49

66 Bottini 1991, 97. Al medesimo gruppo vanno ascritti, oltre agli esemplari da Eboli (loc. Santa Croce, tomba 40), Poseidonia (loc. Gaudo, tomba 164) e Lavello (loc. Cimitero, tomba 686), già citati (supra ntt. 51 e 57), ad es., anche quelli della collezione statunitense Leon Levy (Cahn, 1990: 114 n. 95 a), da Poseidonia, loc. Porta Aurea, tomba 2 (Pontrandolfo, Rouveret, 1992: 364-365 n. 11), da imprecisata località dell'Italia meridionale (da ultimo, Tagliamonte, 1994 a: 257-258 n. C3) e dal territorio ma-rrucino (Tagliamonte, 2003 b: 156-157, $169 \mathrm{nn}$. N4, N5).

67 Oltre tale limite non permettono, al momento, di risalire, a livello di "Realien", i dati di contesto e associazione relativi agli esemplari più antichi riferibili al Gruppo B1: si vedano, ad es., i materiali inclusi nei corredi che hanno restituito i due elmi da Poseidonia (locc. Gaudo, tomba 164, e Porta Aurea, tomba 2) citati alla nt. precedente. Coerenti con tale indicazione cronologica sono, sempre nel caso di Poseidonia, pure i dati desumibili dalla documentazione iconografica, pur nelle difficoltà di giungere a così puntuali proposte di identificazione dei tipi di elmo lì raffigurati (che sem- ne consentono, come visto ${ }^{65}$, un pressoché certo riferimento al Gruppo B1 della classificazione elaborata da A. Bottini ${ }^{66}$ per siffatti elmi, e, che ne suggeriscono, pertanto, una datazione non anteriore al secondo venticinquennio del IV secolo $^{67}, \mathrm{o}$, più probabilmente, alla metà dello stesso $^{68}$. Ed è proprio a un esemplare simile, o comunque ascrivibile alla classe suditalico-calci-

brano, ad ogni modo, nel complesso, riferibili al modello suditalico-calcidese): cfr., ad es., per quanto riguarda la pittura funeraria, Pontrandolfo, Rouveret, 1992: 42 figg. 27-28, 43 figg. 29-31, 101 fig. 3, 111 fig. 2, ecc.

68 Il profilo anteriore sinuoso del margine anteriore delle paragnatidi raffigurate sui due esemplari da Pietrabbondante richiama infatti, sempre a livello di "Realien", quello che caratterizza gli analoghi paraguance non decorati applicati a elmi suditalico-calcidesi, databili, sulla base dei dati di contesto e associazione, alla seconda metà del IV sec. a.C., per lo più al terzo quarto dello stesso: ad es:, Bottini, 1991: 97 nn. B1, B2, B4, ecc. Nei citati esemplari da Poseidonia (supra nt. 66) riferibili al secondo venticinquennio del IV sec. a.C. la parte inferiore del margine anteriore delle paragnatidi assume piuttosto, almeno nel caso dell'elmo dalla tomba 164 in loc. Gaudo, un andamento rettilineo, che richiama evidentemente quello dei più antichi "Helme mit spitzen Wangenschirmen" del tipo 4 delle classificazioni degli elmi calcidesi proposte da Kunze (1967: 137; 1994: 41 ss.) e Pflug (1988 a: 142-143), e che, peraltro, sussiste ancora nella seconda metà del IV sec. a.C., come dimostra, ad es., l'elmo da Eboli (loc. Santa Croce, tomba 40) summenzionato (supra nt. 51) e, ad abundantiam, la documentazione iconografica. Cfr. al riguardo anche Dintsis, 1986: 138 ss., che in sostanza riporta i tipi Kunze 7/Pflug 5 e Kunze/Pflug 4 all'interno di un unico tipo (il 3: "Helm mit geschweiften, spitz oder rund zulaufenden Wangenklappen”). 

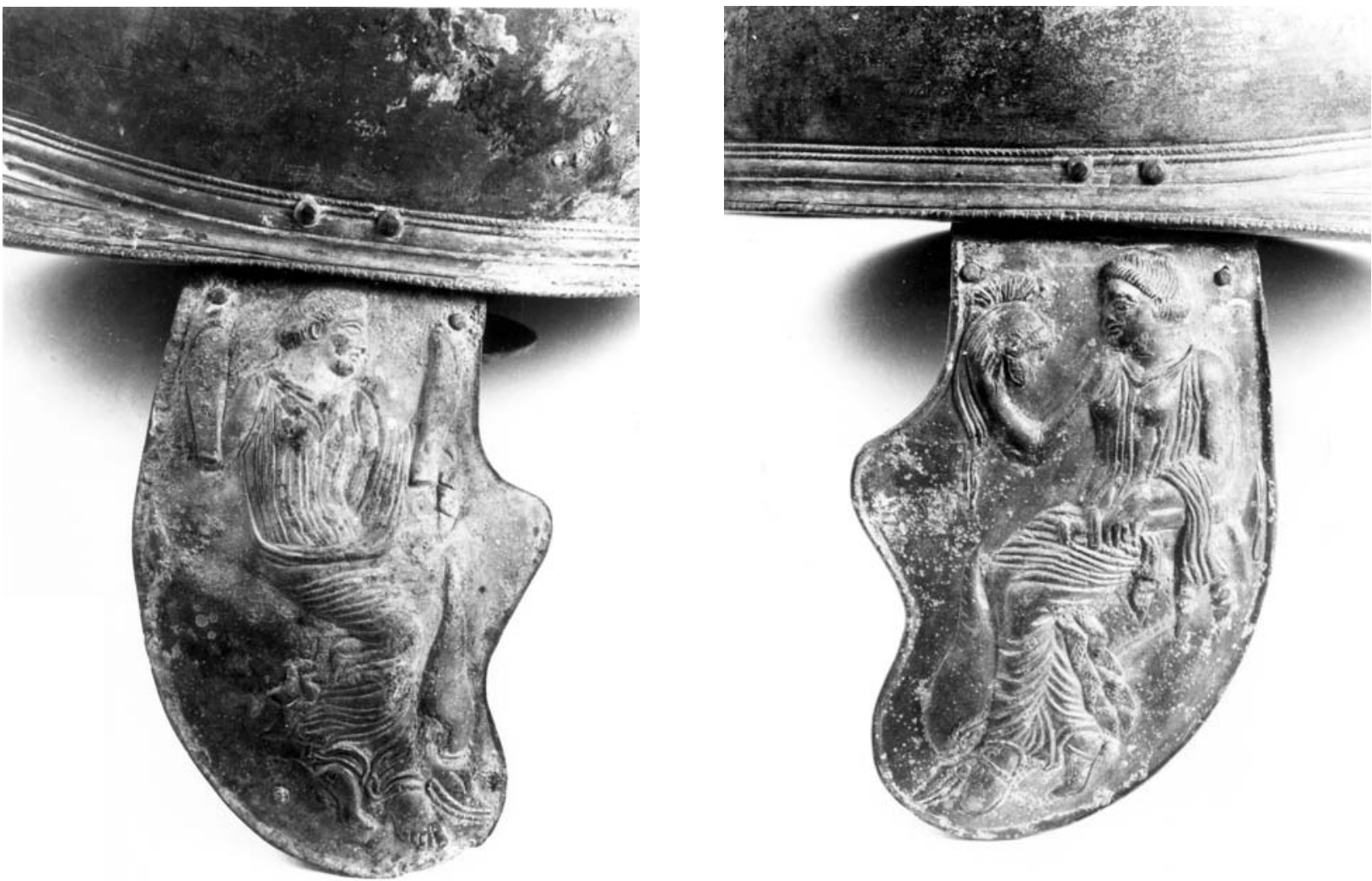

Figg. 16-17 - Museo Archeologico Nazionale di Napoli: particolare delle paragnatidi anatomiche con decorazione figurata a sbalzo dal santuario di Pietrabbondante (Soprintendenza per i Beni Archeologici delle Province di Napoli e Caserta, neg. nn. 69239-69240).

dese, che peraltro vanno attribuite, come sopra ricordato, le due paragnatidi in questione: paraguance anatomici simili, arricchiti da decorazioni figurate di questo genere, non parrebbero, del resto, adottati in rapporto a quelle classi di elmi, per le quali pure è testimoniato, attorno alla fine del V secolo, l'impiego di paragnatidi mobili nel mondo magno-greco e, più in generale, in quello greco $^{69}$. D'altronde, il profilo sinuoso, bilobato, del margine anteriore delle due paragnatidi sem-

69 Come, ad es., nel caso degli esemplari attici e calcidesi, editi o riediti in Pflug, 1989: 63 n. 31, 92 n. 83, 93 n. 85. Paragnatidi mobili decorate a sbalzo con scene di combattimento ha anche l'elmo calcidese in bronzo ageminato in argento dalla tomba di guerriero di Todi, loc. San Raffaele, conservato nel Museo Nazionale Etrusco di Villa Giulia a Roma (Dintsis, 1986: 138 nt. 14, 139 nt. 20, taf. 66.2; Pflug, 1988 a: 150 Abb. 18)

70 Vedi quanto osservato supra $\mathrm{ntt}$. 67-68. In particolare, il profilo delle due paragnatidi decorate da Pietrabbondante richiama, ad es., quello degli analoghi pezzi applicati a elmi suditalico-calcidesi di provenienza canosina, datati, dal contesto, attorno al 330 a.C. (Cahn, 1989: 40-42 n. W 23a, 55-57 n. W 24 a). Vicini anche quelli di alcune paragnatidi associate a elmi "frigi" e "frigio-calcidesi": si veda, ad es., l'esem- brerebbe più vicino a quello documentato in esemplari dell'inoltrato IV secolo ${ }^{70}$, piuttosto che a quelli di epoca precedente (e diverso, come detto, da quello dei pezzi riferibili a elmi a bottone).

Peraltro, un simile slittamento cronologico, dalla fine del V alla seconda metà del IV sec. a.C. era stato già, con buone motivazioni, esplicitamente affermato $^{71}$ in rapporto all' ala con figurazione di Nike, rinvenuta nel corso degli scavi

plare della Collezione Ceccanti di Firenze (Venezia, 1996: 653 n. 330 I). Accogliendo l'ipotesi di un'originaria pertinenza delle due paragnatidi in questione a un elmo di tipo suditalico-calcidese, ci troveremmo di fronte a uno dei non rarissimi casi di richiamo "interno" fra esemplare reale di elmo e sua raffigurazione (o, meglio, raffigurazione di elmo della medesima tipologia) nell'apparato decorativo dello stesso: si confronti al proposito, ad es., proprio l'elmo "frigio-calcidese" della Collezione Ceccanti, appena menzionato, la cui calotta è ornata da protomi maschili recanti elmo di tipo "frigio".

71 Capini, S. in Milano, 1991: 159. Alle considerazioni e ai confronti qui citati, si può aggiungere, per la Nike, il richiamo alle figure di eroti volanti posti ai fianchi di Afrodite nella neck-amphora dalla tomba 13 della 
condotti da A. La Regina, con ogni probabilità pertinente anch'essa, come pure sopra ricordato, a un elmo di tipo suditalico-calcidese. Anche nel caso delle paragnatidi anatomiche decorate con ornati vegetali, le considerazioni e i confronti richiamati in anni relativamente recenti da P.G. $\mathrm{Guzzo}^{72}$, in particolare per quanto riguarda il motivo della palmetta con i petali a volute rivolti tutti nella medesima direzione, sembrerebbero orientare verso una datazione delle stesse a un momento avanzato del IV sec. a.C., piuttosto che agli inizi del secolo, se non alla fine del V, come a suo tempo proposto ${ }^{73}$. A tale datazione meglio si addice, del resto, morfologicamente, il profilo sinuoso, bilobato, più o meno accentuato, del margine anteriore delle paragnatidi stesse ${ }^{74}$.

Delle altre tre paragnatidi, o presunte tali, il frammento recante scena di Amazzonomachia sembrerebbe replicare, con qualche minima differenza, il medesimo schema iconografico riprodotto su una lamina bronzea "dalla Turchia", interpretata da B.T. Aitken come paragnatide di elmo e da D. Cahn forse come spallaccio di corazza, ma da entrambi datata a un momento avanzato del IV sec. a.C., se non agli inizi del $\mathrm{III}^{75}$. Per gli altri due pezzi, considerazioni di carattere iconografico, stilistico e, soprattutto, tipologico-funzionale, stante il loro probabile o possibile riferimento a elmi suditalico-calcidesi, potrebbero in qualche modo giustificare l'ipotesi di un abbassamento della loro datazione al pieno IV sec. a.C., rispetto a quanto in precedenza affermato $^{76}$.

È evidente che tali osservazioni necessitano di

necropoli pestana di Licinella, attribuita, insieme ad altri vasi del corredo, al Pittore di Afrodite: Greco, 1970: 11 ss., tavv. I, III.

72 Guzzo, 1990: 7. Sul motivo della palmetta nella cultura figurativa italiota protoellenistica cfr. Pfrommer, 1982: 120 ss. 122 nt. 3.

73 D'Agostino, 1980: 141, ripreso in Milano, 1991: 157158 nn. d17, d18.

74 Cfr. quanto osservato supra ntt. 67 e 69.

75 Aitken, 1982: 58-60; Cahn, 1989: 24-25 n. W 15.

76 Ciò vale, evidentemente, per la paragnatide con raffigurazione di Eracle, essendo l'altra (quella con guerriero in nudità eroica) inedita. Per tale paragnatide D'Agostino (1980: 142) pensava, essenzialmente sulla base del dato stilistico, a una datazione "al volgere dal V al IV secolo"; tuttavia, gli stessi confronti in tal senso addotti dallo studioso, sembrerebbero in qualche modo ricondurre a un orizzonte cronologico più recente: si vedano, ad es., La Rocca, E., in Roma, 1973: 286288 n. 426; Cerchiai, 1984: 53 ss; Bottini, 1992: 158, per una datazione a un momento avanzato o finale del ulteriori approfondimenti di studio e verifiche, per lo meno nella misura in cui lo consente l'ancora insufficiente grado di conoscenza della bronzistica magno-greca, e di quella tarentina in particolare, ${ }^{77}$, ma certo in modo più persuasivo di quanto sia stato possibile fare in questa sede. Seppure non senza dubbi e incertezze, dei quali del resto bene testimoniano le oscillazioni di inquadramento cronologico relative ad alcuni degli esemplari meglio noti della bronzistica tarantina o presunta tale ${ }^{78}$, quella di una datazione al pieno IV sec. a.C. del gruppo di paragnatidi decorate da Pietrabbondante sembrerebbe essere qualcosa in più di una mera ipotesi di lavoro.

Tali considerazioni portano in sostanza a ridimensionare il dato della supposta esistenza, fra i materiali votivi di Pietrabbondante, di un più antico nucleo di armi databili tra la fine del V e la prima metà del IV a.C., o quanto meno quello di una sua identificazione tout court con il gruppo delle armi decorate appena esaminate. In assoluto, non si può infatti escludere che tra le armi dedicate nel santuario di Pietrabbondante ve ne siano alcune (o, forse, anche altre) databili al periodo in questione, se non prima. Potrebbe essere, ad esempio, questo il caso di alcuni dei ganci di cinturone "sannitici" (Fig. 18) provenienti dagli scavi ottocenteschi e novecenteschi, in particolari di taluni di quelli riferibili ai tipi 2B, 4A, 4B e 1B della classificazione elaborata da $\mathrm{M}$. Suano $^{79}$. Per questi, infatti, le conoscenze sinora acquisite, che ne fissano l'ambito cronologico di attestazione fra la seconda metà del $\mathrm{V}$ e la fine del IV sec. a.C. ${ }^{80}$, potrebbero fare pensare, alme-

IV sec. a.C. della paragnatide con Amazzonomachia da Palestrina. Quest'ultima, peraltro, in anni recenti, è stata avvicinata alle decorazioni a rilievo che contraddistinguono le ceramiche argentare di area etrusca della prima età ellenistica: Guzzi, 2002: 122; Michetti, 2003: 46.

77 Lippolis, 1984; Bottini, 1992; Tarditi, 1996; Morel, 2002: 539 ss.

78 Cfr. supra nt. precedente. Limitatamente al caso delle paragnatidi, si può ricordare come l'esemplare da Palestrina menzionato supra nt. 76 venga considerato da Lippolis, 1984: 40 forse il più antico fra quelli del gruppo includente i "Siris bronzes", laddove Cerchiai, 1984: 56 e Bottini, 1992: 158 lo ritengono il più recente.

79 Suano, 1986: 2-5; 1991: 135-136; 2000, 184-185, 191.

80 Osservazioni al proposito in Suano, 1986: 25-28; 1991: 135-137; Romito, 1995: 16 ss.; Sannibale, 1995: 981 ss.; Romito 2000: 193-194; Suano, 2000: 184-185. 


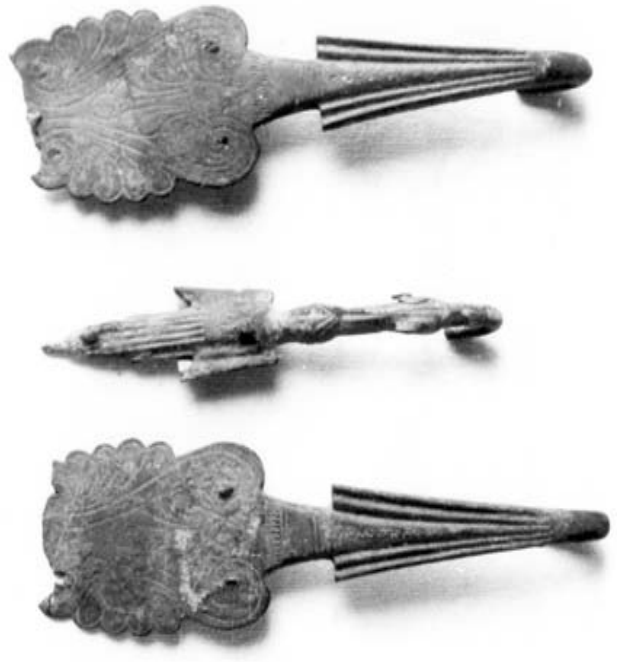

Fig. 18 - Museo Archeologico Nazionale di Napoli: ganci di cinturone "sannitico" dal santuario di Pietrabbondante (Soprintendenza per i Beni Archeologici delle Province di Napoli e Caserta, neg. n. 22073).

no per qualche esemplare ${ }^{81}$, a una datazione ancora al V secolo a.C.; in assenza di ulteriori indicazioni che a concorrano a meglio circoscriverla, è evidente che tale datazione rimane comunque ipotetica e necessariamente generica. Lo stesso parrebbe potersi dire a proposito di alcune delle punte di lancia (Fig. 19) e di giavellotto a lama foliata, più o meno allungata, con innesto a cannone, rinvenute nel santuario ${ }^{82} \mathrm{e}$ che pure potrebbero risalire al $\mathrm{V}$ secolo ${ }^{83}$. Anche in questo caso, allo stato attuale delle conoscenze è difficile avere certezze, dal momento che si tratta di tipi in uso nel lungo periodo, per la cui datazione sono in genere dirimenti dati di associazione e di contesto ${ }^{84}$.

81 Al tipo 2B della classificazione di M. Suano sono riferibili gli esemplari: Romito, 1995: 60-62 nn. 85-97, 99. Al tipo 4A: Romito, 1995: 62-63 nn. 100, 103. Al tipo 4B: Romito, 1995: 62 n. 101. Al tipo 1B: Romito, 1995: 63 nn. 106-108.

82 Per i numerosissimi esemplari di punte di lancia e giavellotto in ferro rinvenute a Pietrabbondante nel corso degli scavi borbonici: Ruggiero, 1888: 617 ss. Di queste, solo otto (punte di lancia) compaiono nel catalogo di Fiorelli, 1869: 8 nn. 70-77. In Isernia, 1980: 153 n. 48 è poi edita una punta di lancia rinvenuta in occasione degli scavi condotti da A. La Regina.

$83 \mathrm{Si}$ veda proprio la punta di lancia pubblicata in Isernia, 1980: 153 n. 48, avvicinabile al tipo 4 della classificazione di Russo, 1988: 248-249, che perdura, comunque, nel corso del IV sec. a.C. (ad es., Melfi, 1993: 183 nn. 2-3).

84 Russo, 1988: 249.

85 Su tale problematica vedi, con riferimento all'ambito italico, in particolare Guzzo, 1990: 8 ss.; 1993: 167-168; Künzl, 1997: 61 ss.; Bottini, 1999: 17 ss.; Tagliamonte,
Ma tornando al nucleo di armi decorate, o meglio di elementi di elmi e forse di altre eventuali parti dell'armamento arricchiti da elaborate e raffinate decorazioni a sbalzo, c'è da aggiungere che in riferimento ad essi si pone poi il problema, sempre difficile da definire in casi come questi, della loro interpretazione in termini funzionali. Si tratta di capire, in altre parole, se siano armi di uso reale o piuttosto "da parata", ovvero armi prevalentemente, se non esclusivamente, destinate all'ostentazione di uno status privilegiato, esibite in contesti sociali di tipo particolare, per lo più in connessione ad eventi e occasioni di carattere rituale e cerimoniale ${ }^{85}$. I confini fra $\mathrm{i}$ due ambiti sono, per la verità, spesso labili, incerti, come ho altrove cercato di dimostrare, evidenziando le valenze assunte, sul piano funzionale -in senso lato-, dalla dimensione estetica dell'armamento ${ }^{86}$. Anche armi riccamente decorate, che per molti versi appaiono definibili o vengono tout court definite "da parata", hanno o possono avere una loro intrinseca funzionalità pratica a fini bellici e, conseguentemente, reale impiego sul campo di battaglia. Né mancano nelle fonti letterarie ed, entro certi limiti, in quelle iconografiche attestazioni e indizi in tal senso. Solo per citare alcuni casi pertinenti all'orizzonte storico e culturale in questione, si possono ricordare al riguardo le indicazioni di cui disponiamo circa le insignia arma Samnitium indossate dai guerrieri schierati nella legio linteata sannitica opposta ai Romani nella battaglia di Aquilonia nel 293 a.C. ${ }^{87}$, o il riferimento allo splendido armamento che, tra i suoi soldati e i nemici, rende riconoscibile - ma anche più vulnerabile Pirro $^{88}$. E gli esempi si potrebbero moltiplicare ${ }^{89}$.

1999: 113-114; Guzzo, 2001: 24-26; Tagliamonte, 2003 a.

$86 \mathrm{Nel}$ caso specifico, di quello arcaico delle popolazioni di area picena e "medio-adriatica": Tagliamonte, 1999 e 2003 a.

87 Armi, che, come afferma Livio 10.39.13, "più che servire loro (scil. i Sanniti), erano state delle belle spoglie per il nemico vittorioso" (... spoliaque ea honestiora victori hosti quam ipsis arma fuisse).

88 Come attesta il vano attentato portato al re epirota dal frentano Oblakos in occasione della battaglia di Eraclea del 280 a.C.: sull'episodio cfr. le versioni di Dio. Hal. 19.12; Plut., Pyrrh. 16.12-16; Flor. 1.13.7; Iord., Rom. 154.

89 Estendendosi, entro certi termini, anche alla documentazione iconografica, seppure in riferimento a contesti segnati da elevata ideologizzazione e forti istanze autocelebrative: vedi, ad es., lo splendido armamento esibito dal cavaliere ritratto in una delle lastre dipinte della tomba di via Seminario, a Nola: da ultima, Benassai, 2001: 95-97 n. N. 4 (con rinvii alla bibliografia). 

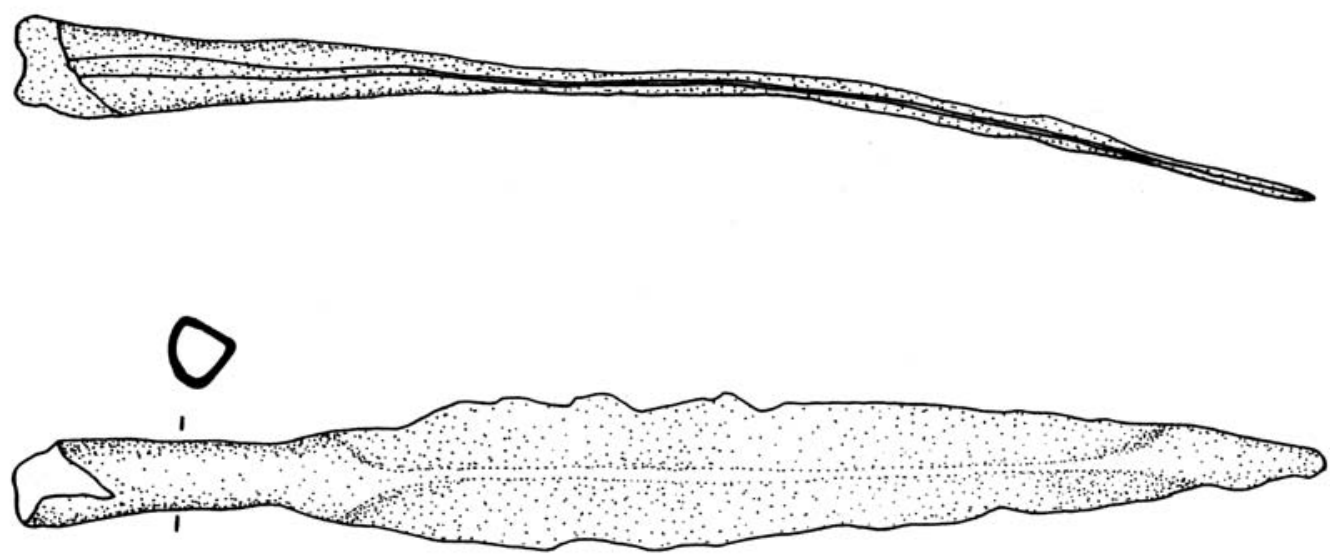

Fig. 19 - Soprintendenza per i Beni Archeologici del Molise, depositi: punta di lancia (disegno) dal santuario di Pietrabbondante (da Isernia, 1980: tav. 43 fig. 41.48).

Sulla base di tali presupposti, è evidente che l'eventuale definizione dell'arma stessa come arma "da parata" poggia di per sé non tanto sulla valutazione della ricchezza e del livello qualitativo dell'apparato decorativo che la contraddistingue quanto piuttosto su quella delle caratteristiche morfologiche, dimensionali e materiche che le sono proprie. Laddove queste ultime rivelino scarsa o nulla efficacia in termini di intrinseca funzionalità difensiva $(o$, più raramente, offensiva) e ad esse si associno decorazioni di particolare pregio, che ne fanno in pratica delle insegne di rango, si potrà ragionevolmente supporre che le armi in questione costituiscano degli esemplari "da parata", acquisiti ed esibiti come beni di prestigio e simbolo di status.

Ora, nel caso specifico degli elmi cui erano riferibili le paragnatidi e l'ala decorate sopra esaminate, non abbiamo elementi decisivi per potere affermare che sussistano tali condizioni. Lo spessore della lamina dalla quale è ricavata ciascuna delle due paragnatidi decorate, associate all'elmo "a bottone" di Napoli, o quello delle due lamine, che, unite, costituiscono ciascuna delle due paragnatidi, pure anatomiche, con ornati vegetali del Museo di Campobasso non parreb-

90 Ancorché sia di certo ipotizzabile una maggiore resistenza delle paragnatidi anatomiche realizzate in bronzo massiccio.

91 Capini, S. in Milano, 1991: 158-159 n. d21. Da non escludere del tutto, sebbene improbabile, l'ipotesi che gli interventi di restauro/riparazione dell'ala, di certo più fragile rispetto ad altri pezzi in bronzo come le paragnatidi, possano essere stati determinati dalle condizioni di esposizione e/o di conservazione della stessa, una volta che questa fosse stata dedicata nel santuario.

92 Come attestano chiaramente almeno due delle parag- bero differire in modo significativo da quello noto per paragnatidi anatomiche non decorate, pertinenti a elmi di tipo suditalico-calcidese, risultando pertanto analoga la loro intrinseca funzionalità protettiva ${ }^{90}$. Nei restanti casi, è la sola lamina anteriore a essere conservata, sicché delle tre paragnatidi (o presunte tali) è difficile valutare la reale efficacia difensiva. Da una sola lamina, ma questo è normale, è pure costituita l'ala decorata con figura di Nike. Su quest'ultima sono stati anzi notati interventi di restauro antico, che farebbero pensare a un reale uso bellico dell'elmo sul quale essa era apposta ${ }^{91}$. Anche la presenza di fori o ganci posti sul margine inferiore delle paragnatidi decorate ${ }^{92} \mathrm{e}$ utili ad assicurare il fissaggio del laccio di chiusura o del sottogola sembrerebbe andare nella medesima direzione, quantunque essa non sia riscontrabile su tutti gli esemplari in questione ${ }^{93}$.

In effetti, a ben vedere, si ha motivo di credere che quello della pertinenza o meno delle paragnatidi decorate da Pietrabbondante ad armi "da parata" rappresenti, tutto sommato, un falso problema. Più che pezzi riferibili ad armi "da parata", nelle nostre paragnatidi vanno piuttosto riconosciuti gli elementi che in misura maggiore,

natidi anatomiche decorate con ornati vegetali (gli esemplari inv. nn. 4434 e quelo edito in Roma, 2000: 45 fig. 52: cfr. supra nt. 47) e, a quanto sembra, il frammento di paragnatide con scena di Amazzonomachia (cfr. supra nt. 44). Lo stato lacunoso delle altre due lamine sopra menzionate (cfr. supra ntt. 43, 45) non consente né di accertare né di escludere la presenza di fori o ganci sul margine inferiore delle stesse.

93 Sulle paragnatidi con figure di Nereidi del Museo Archeologico Nazionale di Napoli non parrebbe, infatti, esservene traccia: ciò potrebbe, pertanto, essere indi- 
se non esclusiva, concorrevano alla decorazione degli elmi ${ }^{94}$ ed, eventualmente, alla loro qualificazione come armi “da parata". A tale affermazione sembra condurre, peraltro, anche la constatazione del fatto che tutte le paragnatidi e gli altri pezzi decorati riferibili al gruppo che include i cosiddetti "Siris bronzes" sono stati trovati disgiunti dagli elmi e/o dalle altre parti dell'armamento difensivo cui erano o dovevano essere in origine pertinenti ${ }^{95}$, circostanza questa che sì non appare esclusiva ${ }^{96}$, ma che ben difficilmente si potrà considerare casuale. Su tali basi sembra, pertanto, di potere affermare che con tutta probabilità le paragnatidi anatomiche a decorazione figurata circolassero separatamente, o perlomeno anche separatamente dagli elmi (suditalico-calcidesi e, forse, calcidesi) cui dovevano o potevano essere associate, conferendo loro pregio e prestigio $^{97}$. E peraltro possibile che esse sostituissero,

zio di una scarsa funzionalità pratica dei due paraguance. Su una di esse, la destra, si nota tuttavia, al di sotto della figura di Nereide con schinieri, la presenza di quella che sembrerebbe essere la testa di un ribattino. $\mathrm{Al}$ momento di redigere questo testo per la pubblicazione, non è stato però possibile effettuare un controllo autoptico sui pezzi, essendo questi esposti a Benevento, nella mostra di cui supra nt. 27. Occorre, inoltre, tenere conto del fatto che i due reperti sono stati oggetto di restauri ottocenteschi e che non sempre fori e/o ganci sono presenti in esemplari non decorati, per i quali non si ha motivo di dubitare del loro reale uso bellico. D'altro canto, la presenza di fori in paragnatidi anatomiche con decorazioni figurate a sbalzo è comunque documentata: ad es., sugli esemplari editi da Cahn, 1989: 24 n. W 14; Pflug, 1989: 93 n. 84; Cahn, 1990: 114 n. 95 a,117 figg. 1-3. Da ricordare, infine, a proposito della effettiva utilizzazione in contesti bellici degli elmi dedicati a Pietrabbondante, che Ruggiero, 1888: 631 rilevò su uno degli elmi "a bottone" evidenti tracce di colpi di fendente.

94 Se l'elmo dalla tomba 686 di Lavello (cfr. Bottini, Fresa, 1991: 65 n. $31 *$ ), nel suo ottimo stato di conservazione, ci restituisce un esempio eccezionalmente completo della decorazione "accessoria" che contraddistingueva gli elmi di tipo suditalico-calcidese (crista, alae, pinnae) e se quello della collezione statunitense Leon Levy (Cahn, 1990: 114 n. 95 a) ci offre una straordinaria testimonianza del livello qualitativo e delle ricchezza dell'apparato decorativo (a sbalzo e a incisione) della calotta, in genere sono proprio le paragnatidi con figurazioni a sbalzo gli elementi nei quali la dimensione estetica dell'armamento trova espressione. Al di fuori di quelle consuete, di tipo strutturale, riferite al frontale e alle spirali temporali degli elmi suditalico-calcidesi, risultano infatti estremamente rare altre forme di ornamentazione a decorazione sbalzata della calotta: si vedano, ad es., la protome gorgonica presente sul frontale dell'esemplare "da Capodignano” (Bottini, 1991: 97 anche in via temporanea, le paragnatidi effettivamente utilizzate in guerra, per lo meno in quelle occasioni di carattere cerimoniale che prevedevano l'ostentazione di armi "da parata" o in quelle circostanze che comunque potevano rappresentare un momento di verifica di status all'interno della comunità ${ }^{98}$.

La loro presenza tra gli ex voto dei santuari, certa per Pietrabbondante e Dodona ${ }^{99}$, oltre a individuare il valore di beni di prestigio da esse assunto, sembrerebbe rispondere a quella logica del dono della pars pro toto, della quale, almeno nel caso Pietrabbondante, abbiamo così tanti esempi nelle dediche delle numerose e isolate paragnatidi anatomiche e, soprattutto, a tre dischi rinvenute nel corso delle esplorazioni condotte a Calcatello. Come mostrano chiaramente $i$ fori presenti in diversi esemplari ${ }^{100}$, almeno una parte di esse doveva essere affissa alla trabeazio-

n. B 3) o la figura di cane su di un frammento della parte posteriore della calotta di un esemplare di ignota provenienza (Berlin, 1988: 436 n. 47). Un'applique ovale, oggi perduta, era posta al centro del frontale del summenzionato elmo della collezione Leon Levy.

95 Cfr. in tal senso l'elenco riportato in Aitken, 1982: 61.

96 Come dimostra, proprio nel caso del santuario di Pietrabbondante, la presenza di numerose paragnatidi anatomiche e a tre dischi non decorate, rinvenute (e presumibilmente dedicate) separate dagli elmi cui dovevano essere in origine associate, ancorché non sia da escludere in qualche caso (perlomeno, in quelli in cui i paraguance non recano fori di affissione) l'ipotesi di un loro eventuale riferimento a qualcuno di quei frammentari esemplari di elmo, menzionati nei resoconti ottocenteschi degli scavi borbonici: supra nt. 28.

97 Cfr. quanto osservato supra nt. 93.

98 Sui fenomeni di sostituzione e di recupero/reimpiego a scopo bellico di paragnatidi, relativamente però agli elmi "a bottone", vedi, ad es., la casistica individuata, su basi archeologiche, da Vitali, 1992: 292, 359, 377.

99 Aitken, 1982: 61.

100 Praticamente irrilevante negli esemplari di paragnatidi (decorate e non) conservate nel Museo Archeologico Nazionale di Napoli, la presenza di fori di affissione risulta invece comune su quelle custodite in quello di Campobasso. Nel corso degli ultimi anni, una maggiore attenzione al dato contestuale ha sollecitato, relativamente ai santuari italici (specie, quelli lucani) che hanno restituito armi, una serie di osservazioni circa le presunte modalità di dedica e di esposizione/conservazione delle stesse: vedi ad es., Adamesteanu, Dilthey, 1992: 69-70; Nava, Poccetti, 2001: 96-97 (Rossano di Vaglio); Greco, 1991: 79-80; Bruscella, E. in Nava, Osanna, 2001: 100-101 (Torre di Satriano); Russo, 1995: 56 (Armento, loc. Serra Lustrante); La Torre, 2002: 337338 (Campora San Giovanni, loc. Imbelli); Nava, M.L. in Taranto, 2004: 980 (Civita di Tricarico). 
ne lignea di alcuni degli edifici del complesso santuariale ("tempio ionico", portici?), mentre un'altra poteva essere forse compresa nella congeries armorum, di cui si è ipotizzata l'erezione nella spianata antistante il tempio A. Le paragnatidi con decorazione figurata, o per lo meno alcune di esse, non furono affisse, dal momento che su di esse non parrebbero ravvisarvi tracce di fori ${ }^{101}$. È possibile che tali paragnatidi, siano esse da considerarsi preda di guerra (skỳla o làphyra apò tòn polemìon) o armi proprie (òpla oìs autòs echreito), siano frutto di dediche collettive o da parte di singoli. Tenuto conto dei caratteri di omogeneità che a più livelli sembrano caratterizzare questo gruppo di bronzi sbalzati e della sostanziale coerenza del discorso narrativo che le loro figurazioni compongono, c'è da tuttavia chiedersi se esse non possano avere costituito un insieme unitario (sia esso riconducibile a un trofeo o a un donario) ${ }^{102}$, peraltro presumibilmente riferibile alle divinità titolari del culto nel santuario pentro ${ }^{103}$.

In questo secondo caso ci troveremmo di fronte alla possibilità, in qualche modo già adombrata, di una acquisizione, e quindi di una successiva dedica, delle paragnatidi decorate attraverso canali diversi da quello della preda bellica. In tal senso, considerata anche la complessiva proposta qui formulata di un inquadramento cronologico del gruppo all'avanzato IV secolo a.C., difficilmente si può sfuggire alla suggestione di richiamarsi a quei rapporti che, soprattutto a partire dall'epoca delle strategie di Archita (367-361 a.C.), il mondo sannita venne intrattenendo, a più livelli, ma specialmente su quello della colla-

101 Fori di affissione sono certamente presenti su due delle paragnatidi con decorazione a ornati vegetali (inv. nn. 4433, 4434: cfr. supra nt. 47). Risultano invece assenti (ma vedi supra nt. 93) nelle due paragnatidi con figure di Nereidi del Museo di Napoli (cfr. supra nt. 41) e, a quanto sembra, nell'ala con raffigurazione di Nike (cfr. supra nt. 46). Più incerti i restanti casi, anche se per due di essi (cfr. supra ntt. 43, 45) l'integrità della parte centrale della lamina (ovvero, della zona nella quale viene in genere praticato il foro di affissione) parrebbe fare escludere tale eventualità.

102 A ciò non osta, in entrambi i casi, né il dato (di scavo) della provenienza dei pezzi in questione da diversi punti dell'area sacra (stanti il comune fenomeno della dispersione degli originari contesti di dedica in ambito santuariale e le specifiche indicazioni delle quali in tal senso disponiamo per Pietrabbondante) né quello di un'eventuale sfasatura cronologica fra i pezzi considerati (risultando questa improbabile e potendosi, comunque, ipotizzare che il pezzo più recente costituisca il terminus ante quem non per l'erezione del trofeo o la dedica del donario, sia esso pubblico o privato). borazione militare, con Taranto; ovvero proprio con quella pòlis, nella quale si è visto il probabile centro di produzione delle paragnatidi decorate, o perlomeno di una parte di esse, ancorché tale attribuzione sia al momento più presupposta che provata $^{104}$. Alla luce di tali rapporti, dei quali abbiamo esplicite testimonianze nelle fonti letterarie e significativi riflessi nella documentazione archeologica e numismatica ${ }^{105}$, non sarebbe azzardato ipotizzare che le paragnatidi in questione siano state acquisite da elementi di origine sannitica venuti a contatto con quel mondo, attraverso vie e meccanismi di scambio che certo non è facile specificare e che potrebbero contemplare rapporti di natura commerciale, doni cerimoniali, legami di xenìa, forme di compenso per prestazioni mercenarie, ecc. ${ }^{106} \mathrm{E}$ che, pertanto, esse siano state da questi successivamente dedicate nel santuario di Pietrabbondante come doni o ex voto di particolare pregio e di forte valenza simbolica, indirizzati alle divinità guerriere qui venerate ${ }^{107}$. Dire, poi, nello specifico se tali offerte siano frutto di atti individuali o collettivi di devozione è francamente difficile, per non dire impossibile, in assenza di esplicite indicazioni epigrafiche.

Ad ogni modo, che armi, siano esse preda di guerra (skỳla o làphyra apò tòn polemion) o armi proprie (ópla oìs autòs echreito), fossero dedicate nel corso del IV sec. a.C. in santuari dell'Italia meridionale da singoli elementi di origine sannitica o loro duces lo dimostra esplicitamente un ristretto ma significativo nucleo di iscrizioni in lingua osco-sannita incise su elmi bronzei, sulle

103 Cfr. infra nt. 106.

104 Considerazioni in tal senso in Lippolis, 1984: 40; Bottini, 1992: 158.

105 Su tali rapporti, vedi da ultimi, Mahé, 1999; Mele, 2000; 2002, 94 ss., con rinvii alla bibliografia precedente.

106 Del resto, in questa direzione sembrano andare D’Agostino, B., in Isernia, 1984: 44; Tagliamonte 1989: 525, Colonna, 1996: 46.

107 Sulla possibile identificazione delle divinità (Afrodite Nikephòros/Vacuna, Eracle, Dioscuri) oggetto di culto nel santuario (e, poi, nello specifico, nel tempio B) vedi le osservazioni di Colonna, 1996: 121-125. Proprio la ricorrente presenza delle immagini di Eracle, Nike e dei Dioscuri sulle paragnatidi e sull'ala di elmi in questione, nonché su alcuni ganci di cinturone "sannitici" ascrivibili ai tipi $6 \mathrm{~A}$ e $6 \mathrm{~B}$ della classificazione di M. Suano e pure dedicati nel santuario (vedi infra nt. 164), costituisce una delle argomentazioni sulle quali G. Colonna fonda la propria proposta di identificazione delle divinità titolari del culto. 
quali in più circostanze ho in passato richiamato l'attenzione ${ }^{108}$. Proprio su tali testimonianze epigrafiche, peraltro, ci si è indirettamente basati ${ }^{109}$ per evidenziare, nell'ambito della complessiva interpretazione che individua nelle armi deposte nel santuario di Pietrabbondante degli spolia hostium, il carattere collettivo e "pubblico" di tali dediche, comprovato per l'appunto dall'assenza di iscrizioni di dedica individuale. In realtà, tale assenza non prova nulla: e ciò semplicemente perché in quell'orizzonte cronologico di pieno IV sec. a.C., cui, nell'insieme, vanno riferite le armi dedicate a Pietrabbondante, o la gran parte di esse, nel Sannio pentro non c'è epigrafia ${ }^{110} o$, per lo meno, mancano ancora un uso e una cultura epigrafica che possano considerarsi tali, stando a quanto sinora noto. Quelli delle summenzionate iscrizioni di dedica su elmi rappresentano casi particolari, riconducibili a specifici fenomeni di acculturazione e assimilazione in senso greco, verosimilmente legati ad attività ed esperienze di mercenariato svolte dai dedicanti al soldo degli Italioti ${ }^{111}$. Il silenzio dell'epigrafia non potrà pertanto essere considerato elemento probante ai fini di una univoca interpretazione delle armi rinvenute a Pietrabbondante come frutto di dedica collettiva della preda bellica, ancorché tale regime d'offerta sia presumibilmente quello prevalente.

Sulla base di queste considerazioni e di quelle sopra espresse, si fa dunque strada la possibilità che le armi, almeno in una qualche misura e occasione, possano essere state dedicate anche dagli stessi Sanniti, e non necessariamente in quanto skỳla o làphyra apò tòn polemion. C'è da chiedersi, a questo punto, se al di là del caso delle armi decorate, ci sia una qualche altra possibilità di riscontro nella documentazione in nostro possesso, circostanza questa che parrebbe esclusa da La Regina ${ }^{112}$. Ora, che a Pietrabbondante esista una dimensione anche privata della dedica, ancorché questa occupi uno spazio minoritario rispetto al prevalente carattere collettivo del regi-

108 Tagliamonte, 1989-90: 519-525; 1994 a: 167-172. Cfr. anche Poccetti, 2001.

109 La Regina, 1984: 23.

110 Nel senso indicato da Panciera, 1998: 314.

111 Cfr. supra nt. 108.

112 La Regina, 1984: 23.

113 Per un quadro complessivo dei materiali dedicati nel santuario di Pietrabbondante: Isernia, 1980: 139-161, 172185; Milano, 1991: 157-159.

114 Oltre che nei contributi citati supra nt. 52, indicazioni in tal senso, ad es., in Feugère, 1993: 83; 1994: 11 ss.; Quesada Sanz, 1997: 157 ss.; cfr. Zhmodikov, 2000.

115 La Regina, 1984: 24; 1990: 61. me dell'offerta, è fatto indubbio, che trova esplicita attestazione nei materiali votivi rinvenuti nel santuario, tanto in riferimento a orizzonti più antichi quanto più recenti di quello di pieno IV secolo fin qui richiamato ${ }^{113}$. Che tale dimensione si estenda poi anche alle dediche di armi è, come abbiamo visto e vedremo, una possibilità che andrà verificata nella misura in cui la natura delle evidenze archeologiche lo consente.

A una sia pure non approfondita lettura della documentazione nota, in effetti non emergono elementi per potere escludere che almeno in qualche caso ci si trovi di fronte non a spolia hostium, ma ad armi proprie, appartenute al singolo guerriero sannita e da questo, presumibilmente, dedicate. A ben guardare le armi per le quali si può davvero ritenere alquanto improbabile l'ipotesi di un riferimento all'armamento locale, sannitico, sono proprio gli elmi "a bottone" e le paragnatidi ad essi pertinenti (anatomiche e a tre dischi). Sulla base delle scarse conoscenze che sinora abbiamo dell'armamento dell'esercito romano di età medio-repubblicana, si può infatti asserire che questi furono adottati dalle truppe romane, assumendo peraltro un forte valore connotativo ${ }^{114}$. Come già acutamente osservato da La Regina $^{115}$, tali elmi e le relative paragnatidi andranno, pertanto, in prima istanza riferiti all'armamento dei milites romani, ivi compresi quei cives sine suffragio e socii di origine italica (Campani, Marsi, Peligni, ecc.) schierati nell'esercito di Roma. Nella loro presenza nel santuario molisano si dovrà in effetti cogliere il riflesso di avvenimenti bellici, verosimilmente verificatisi nel corso della seconda e terza guerra sannitica e/o, soprattutto, dei successivi eventi, sino al fallimento della spedizione italiana di Pirro, a seguito dei quali tali armi, sottratte ai Romani e ai loro alleati, furono lì dedicate come preda di guerra ${ }^{116}$.

Ciò detto, va rilevato che per tutti gli altri tipi di armi, difensive e offensive, rinvenute nel santuario non sussistono, al momento, elementi che

116 Significativa appare in tal senso l'assenza di elmi "a bottone" in sepolture di area propriamente sannitica (a fronte di una loro rilevante presenza nelle necropoli di Pretuzi, Marsi, Marrucini, ecc., ovvero di quelle popolazioni che dei Romani furono, di certo, socii ben più affidabili dei Sanniti). Non a caso le poche attestazioni che di elmi "a bottone" si hanno per il territorio sannitico (peraltro, riferibili a esemplari dei quali si ignora lo specifico contesto di rinvenimento) sono più tarde e riguardano, comunque, località (Ruviano, nell'agro di Caiatia; Reino, nel Beneventano) ormai pienamente soggette al controllo romano: riferimenti in Tagliamonte, 2003 b: 161 nt. 111. 


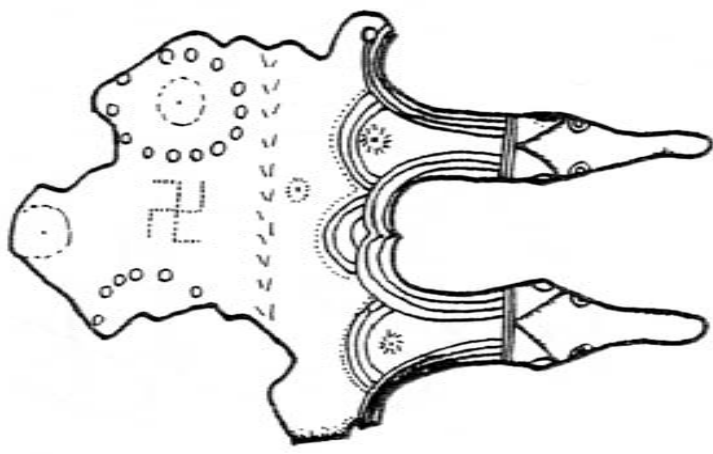

Fig. 20 - Soprintendenza per i Beni Archeologici del Molise, depositi: estremità di cinturone "sannitico" con ganci saldati alla lamina (disegno) dal santuario di Pietrabbondante (da Isernia, 1980: tav. 43 fig. 41.39).

consentano con sicurezza di riferirle all'armamento romano, e in definitiva di considerale $s p o^{-}$ lia hostium. Di certo alcune di esse, come ad esempio, gli schinieri anatomici possono essere più probabilmente ritenute frutto del bottino di guerra piuttosto che parte dell'armamento locale, sannitico, tenuto conto della loro assenza nei contesti funerari pentri del periodo. Tuttavia, diverse indicazioni ricaviamo dalle fonti letterarie, dal momento che queste ${ }^{117}$ ci attestano un uso militare degli schinieri, sia pure particolare, da parte dei guerrieri sanniti.

In altre circostanze, la tipologia delle armi - è ad esempio il caso delle punte di lancia, di giavellotto, di freccia - non consente particolari illazioni al riguardo, considerate l'ampia diffusione e la scarso grado di caratterizzazione distintiva dei tipi in questione.

In altre ancora, la tipologia delle armi e i dati relativi alla loro distribuzione diatopica lasciano del tutto aperta la possibilità di un loro riferimento all'armamento sannitico o a quello dei loro nemici, romani e non. È soprattutto il caso dei cinturoni di tipo "sannitico", presenti a Pietrabbondante, come detto, con una pluralità di

117 Liv. 9.40.3. Cfr. al riguardo Tagliamonte 1994 b.

118 Simile incertezza sussiste, ad es., per il frammento di lamina di cinturone utilizzato, come acutamente ha osservato La Regina (1989: 399-401), per la nota dedica di Caso Cantovios (CIL I ${ }^{2}$ 5). La Regina (1989: 401), tende, ad ogni modo, a considerare il cinturone come appartenuto allo stesso dedicante.

119 Suano 1986, 3; Romito, 1995: 28; Sannibale, 1995: 942, 984, 991; Romito, 2000: 195; Suano, 2000: 185186.

120 Romito, 1995: 28; 2000: 195; Suano, 2000: 185-186.

121 Si tratta della "picca ricurva", rinvenuta nel "terzo esemplari, integri o frammentari, per lo più riferibili, a quanto sembra, al pieno IV secolo, e alla seconda metà in particolare. Le indicazioni di cui oggi disponiamo in merito alla produzione e alla diffusione dei cinturoni "sannitici" -che, è bene ricordare, sono parte dell'armamento, ma, più in generale, dell'abbigliamento tradizionale italicotra le popolazioni indigene dell'Italia meridionale non permettono di precisare se gli esemplari rinvenuti a Pietrabbondante siano appartenuti a individui di origine locale (pentra) o piuttosto a elementi lucani, dauni, peuceti, ecc, se non a esponenti delle più settentrionali genti sabelliche dell'Abruzzo (Marsi, Peligni, Marrucini, ecc. $)^{118}$. Fermo restando che i cinturoni e i ganci di cinturone documentati nel santuario di Pietrabbondante corrispondono a quelli contemporaneamente attestati nelle necropoli del Sannio pentro, rimane dunque incerto, ancora una volta, se essi siano stati lì dedicati come armi proprie o preda bellica. C'è però almeno un caso in cui il dato tipologico induce a ritenere estremamente probabile la prima ipotesi: è quello dei cinturoni con ganci saldati alla lamina (tipo Suano 8A) ${ }^{119}$, dei quali il santuario pentro ha restituito almeno un'attestazione (Fig. 20). Questi infatti trovano al momento la loro area di massima diffusione e, presumibilmente, di produzione in ambiente sangritano $^{120}$, ovvero in un ambito territoriale che, in quell'orizzonte di seconda metà del IV secolo in cui essi paiono in sostanza collocarsi, risulta suddiviso fra Pentri e Carricini. Del resto, l'offerta di cinturoni "sannitici", come vedremo, è fenomeno abbastanza comune in contesti santuariali sannitici, che tuttavia non hanno certo quelle valenze politiche ed etniche proprie di Pietrabbondante.

Questioni aperte e difficili da decifrare nei loro effettivi contenuti sono poi quelle relative alla presenza, tra i materiali di Pietrabbondante, di particolari armi offensive in ferro: un'isolata arma d'asta a lama ricurva e alcune spade lunghe di tipo "celtico". Per quanto riguarda la prima $^{121}$, si tratta di un'arma a un solo taglio

cavamento" il 4 giugno 1858 (Ruggiero, 1880: 631) e catalogata da Fiorelli, 1869: 8 n. 79. Lisolamento del pezzo potrebbe essere in qualche modo ridimensionato (ma non dal punto di vista tipologico) qualora si attribuisse funzione anche militare alla "ronca", lunga 0,95 palmi, recuperata nel medesimo luogo e giorno (Ruggiero, 1880: 631) e per la quale va prioritariamente ipotizzato un originario impiego come attrezzo agricolo. Inoltre, il 3 ottobre 1857, in un'area non troppo distante, quella del "secondo cavamento", erano state riportate alla luce 4 picche, fra le quali "un avanzo di lama più larga delle precedenti, curva e lunga pal. 0,60" (Ruggiero, 1880: 617). 
(lunga $55 \mathrm{~cm}$ circa), con innesto a cannone per l'asta lignea, alla cui estremità essa era posta ${ }^{122}$ (Fig. 3). Per tale esemplare, che sembra restare sostanzialmente senza confronti, per lo meno in ambito osco-sannita e osco-campano ${ }^{123}$, non parrebbero sussistere i presupposti di un possibile riferimento alle teretes aclydes, menzionate in contesti poetici come armi caratteristiche delle genti osche ${ }^{124}$. A livello di “Realien”, il richiamo ai cosiddetti drèpana, attestati in sepolture arcaiche di area enotria ${ }^{125}$, rappresenta al momento l'unico, ma non congruo (per tipologia e funzione), termine di confronto.

Tanto negli scavi ottocenteschi quanto in quelli novecenteschi furono poi rinvenute diverse spade lunghe $(70-75 \mathrm{~cm})^{126}$, con immanicatura a codolo, spalla obliqua, lama a doppio taglio e a punta (Figg. 21-22), caratteristiche che ne consentono un'attribuzione al tipo lateniano, peraltro attestato in contesti funerari e, forse, votivi di area medio-adriatica (picena e abruzzese) della fine del IV e degli inizi del III sec. a.C. ${ }^{127}$. Se il dato cronologico ancora una volta ci riconduce all'epoca delle guerre sannitiche, presumibilmente a una fase avanzata delle stesse, e agli avvenimenti successivi, quello relativo alla loro distribuzione diatopica, al di là del riferimento, un po'

122 Più difficile pensare, per la lunghezza della lama, a un manico.

123 Cianfarani, V. in Cianfarani, Franchi dell'Orto, La Regina, 1978: 200, nt. 50, richiama a confronto armi ossidionali del tipo delle murales falces note dalle fonti letterarie e iconografiche (Reinach, S., in Dict. Ant. II, 2, Paris 1896: 968-971, s.v. falx, con fig. 2873 a p. 970), nonché da qualche esemplare reale (cui la nostra parrebbe in qualche modo avvicinarsi per forma e dimensioni), ma non in ambiente italico preromano. Va, peraltro, detto che, sul versante greco, l'hàrpe viene considerata attributo di divinità ed eroi, come Eracle (ad es., Kokkorou-Alewras, G., in LIMC V.1, 1990, s.v. Herakles: 36 nn. 2000-2004, 2006). Per quanto riguarda quest'ultimo, ne abbiamo, una testimonianza indiretta sul versante etrusco-italico, ad es., nella raffigurazione caricaturale di un pigmeo, definito pater poimilionom ("padre dei nani"), ritratto con leontè, clava e lunga hàrpe nel fregio una cista prenestina del Musée des Beaux Arts di Lione (Bordenache Battaglia, 1979: 108-111 n. 27, tav. CXXXII 27c, CXXXIII 27e; una hàrpe compare, anche, fra le mani di uno dei personaggi protagonisti della scena di caccia riprodotta sul coperchio della cista Ficoroni: Bordenache Battaglia, Emiliozzi, 1990: 211-226 n. 68, tavv. CCCI 68g, CCCIV 68m).

124 Verg., Aen. 7.730; cfr. Serv., ad Aen. 7.730-731. Vedi anche Sil. Pun. 8.550. Né tanto meno appare possibile pensare, trattandosi presumibilmente di un'arma d'asta, ai falcati enses, ovvero alle spade a lama ricurva, che Virgilio (Aen. 7.732) pure attribuisce all'armamento bar- scontato, alla possibile penetrazione di elementi celtici $^{128}$, parrebbe piuttosto orientare a considerarle, specie per quanto attiene alle attestazioni abruzzesi, parte dell'armamento dei socii italici di Roma, per lo meno di quelli più fidati ${ }^{129}$; e, quin$\mathrm{di}$, in sostanza a spiegare la loro presenza nel santuario pentro in quanto spolia hostium. In tal senso è tuttavia abbastanza imbarazzante constatare come praticamente in tutti i casi documentati a Pietrabbondante, o quasi, le spade in questione siano state rinvenute all'interno dei loro rispettivi foderi in lamina di ferro, circostanza questa davvero singolare (quantunque non impossibile da spiegarsi), se si ipotizza che esse siano preda di guerra.

Le considerazioni sin qui svolte evidenziano, dunque, la complessità e problematicità della tematica in questione, stanti le incertezze relative alla produzione e alla circolazione di armi nell'Italia antica ${ }^{130}$ e le ancora troppo scarse conoscenze sull'armamento stesso dell'esercito romano di età medio-repubblicana ${ }^{131}$. Sembra, ad ogni modo, farsi strada la possibilità o, in qualche caso, l'alta probabilità che quelle dedicate nel santuario di Pietrabbondante siano anche armi proprie, appartenute a guerrieri sanniti, e non solo prede di guerra, come affermato in modo

barico e primordiale delle genti osche della Campania. 125 Sui drèpana e sul loro possibile impiego come arma offensiva (da cavalleria, ma non solo) vedi da ultima Russo Tagliente, A., in Berlingò, Russo Tagliente, 19921993: 320, con discussione della problematica e rinvii alla bibliografia precedente. Cfr. anche Johannowsky, 2004: 305 nt. 20.

126 Fiorelli, 1869: 8-9 nn. 87-91; Isernia, 1980: 153 nn. 46, 47.

127 Si vedano, ad es., per quanto riguarda l'Abruzzo, gli esemplari da Sulmona (Van Wonterghem, 1984: $286 \mathrm{n}$. 202 b) Manoppello (D'Ercole, Copersino, 2003: 357) e dal Fucino (Celano, 2003: 95-96 nn. 27-32).

128 Come tali vengono, tradizionalmente, infatti interpretate le sepolture contraddistinte da tale tipo di spada: ad es. Papi, 2000: 152.

129 Cfr. supra nt. 115, quanto annotato a proposito della diffusione degli elmi "a bottone". Non a caso, anche in questa circostanza, le sepolture del Sannio pentro non sembrerebbero, stando per lo meno ai dati sinora noti, restituire esemplari simili di spade.

${ }^{130} \mathrm{Nel}$ corso degli ultimi anni considerazioni al riguardo sono state espresse, per es., da Adam, 1986: 22 ss.; Guzzo, 1990: 7-10; 1992: 28-30; 1993: 167 ss.; Mazzei, 1996: 122-124; Polito, 1998: 21 ss.; Bottini, 1999: 15; Adam, 2000: 130-131.

131 Come emerge, ad es., per citare solo alcuni lavori apparsi in anni relativamente recenti, dalle indicazioni riportate in Feugère, 1993: 75 ss. e Le Bohec, 1997: 14 ss. 
Fig. 21 - Museo Archeologico Nazionale di Napoli: spada lunga di tipo lateniano, con relativo fodero, dal santuario di Pietrabbondante (Soprintendenza per i Beni Archeologici delle Province di Napoli e Caserta, neg. n. 69210).
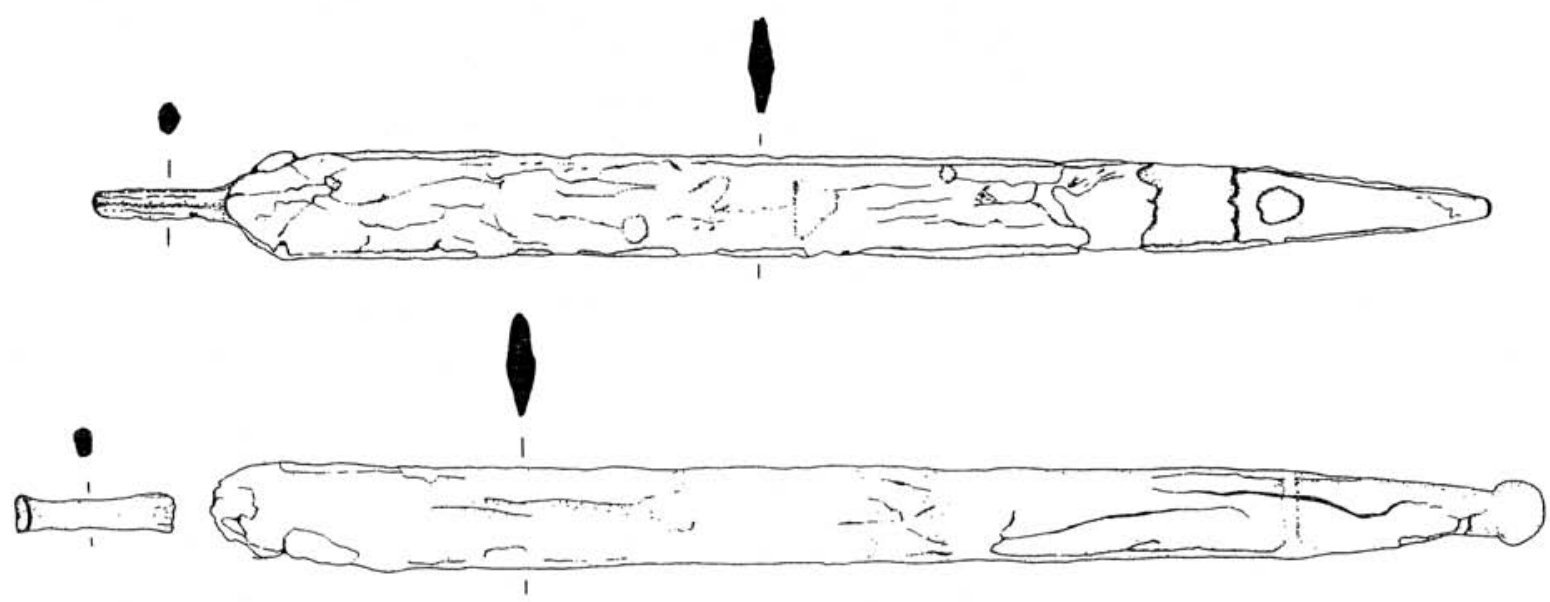

Fig. 22 - Soprintendenza per $i$ Beni Archeologici del Molise, depositi: spade lunghe di tipo lateniano, con relativi foderi (disegno), dal santuario di Pietrabbondante (da Isernia, 1980: 153 fig. 41.46-47).

forse troppo perentorio ${ }^{132}$.

Resta da chiedersi, a questo punto, se indicazioni analoghe provengano o possano provenire da altri santuari e luoghi di culto del mondo sannitico, siano essi a dimensione prettamente locale, cantonale o etnica. La presenza di armi e di altri oggetti ideologicamente riferibili alla sfera bellica nei santuari sannitici dell'epoca sembra, in effetti, un fatto abbastanza ricorrente, anche se mai questo assume la rilevanza e le valenze simboliche e politiche che paiono proprie di Pietrabbondante. Il quadro delle attestazioni, lungi dall'essere irrilevante, è tuttavia, nel suo insieme, certo meno articolato ed eterogeneo rispetto a quanto documentato nell'area sacra di Calcatello. Esso appare, infatti, essenzialmente ristretto a cinturoni e armi offensive.

Le indicazioni più consistenti sono quelle che

132 Cfr. supra nt. 39.

133 Bottini, Rainini, Isnenghi Colazzo, 1976: 496-499 nn. 1181-1199.

134 Romito, 1995: 105 n. 399. riguardano i cinturoni di tipo "sannitico", attestati forse già nel V sec. a.C., ma con più sicurezza nel corso del IV e agli inizi del III sec. a.C. in contesti santuariali di carattere diverso, a valenza etnico-tribale, come nel caso del santuario della Mefite di Valle d'Ansanto, tra gli Irpini ${ }^{133}$; relativi a importanti insediamenti di area caudina (Montesarchio) ${ }^{134}$ e frentana (Larino) ${ }^{135}$; di pertinenza prettamente locale, come quello di San Buono, loc. Fonte San Nicola, sempre fra i Frentani ${ }^{136}$. Accanto a esemplari di cinturoni a fascia in lamina di bronzo integri o in buona misura conservati, la loro presenza è più spesso rivelata dai singoli ganci di chiusura, in misura tale da fare sospettare che, oltre che in termini residuali, essa possa almeno in qualche caso spiegarsi sulla base di quel meccanismo ideologico della dedica della pars pro toto sopra ricordato ${ }^{137}$.

\footnotetext{
135 Isernia, 1980: 308, 311 n. 6.

136 Chieti, 1997: 115 nn. 126-127.

137 Cfr. supra p. 112.
} 
Dal luogo di culto di Fonte San Nicola proviene anche una cuspide di giavellotto in ferro ${ }^{138}$, e armi offensive in ferro (punte di lancia, saurotères) sono state rinvenute in modesta quantità anche in santuari posti nel cuore del Sannio pentro, come quello, a valenza cantonale, di Ercole a Campochiaro $^{139}$. In quest'ultimo caso si registra tra gli ex voto, in particolare quelli rinvenuti nello scarico $\mathrm{B}$, anche la presenza, dal forte valore simbolico, di esemplari miniaturistici di armi offensive (punte di lancia, ascia) ${ }^{140}$. Oltre che nel vicino santuario di Sepino, loc. San Pietro di Cantoni $^{141}$, armi miniaturistiche parrebbero del resto attestate, in maggiori quantità e con più ampia articolazione tipologica, anche agli estremi limiti occidentali di quello che fu in epoca storica il territorio dei Pentri, non lontano da Atina, in un contesto, come quello del santuario di Casalvieri, loc. Pescarola ${ }^{142}$, frequentato già da età arcaica e del quale però ancora troppo poco si conosce al momento. Sempre tra i materiali dello scarico B di Campochiaro, oltre a quella di alcune piccole clave in bronzo riferibili al culto di Eracle, significativa è la presenza di anelli gemini cuspidati e di corone in metallo prezioso.

Se i primi ${ }^{143}$, con ogni probabilità pertinenti alla bardatura equina ${ }^{144}$, attestano verosimilmente la penetrazione nel mondo sannita di quei modelli di autorappresentazione elitaria ispirati ai valori e agli ideali della cavalleria campana, dei quali cogliamo del resto ben più esplicite $\mathrm{e}$ significative testimonianze nella documentazione dell'epoca ${ }^{145}$, le seconde evocano riferimenti ideologici sui quali occorre brevemente soffermarsi. Oltre che nel santuario di Eracle a Campochiaro, dove si sono rinvenuti i resti frammentari di diversi esemplari in oro e argento, per lo più a foglia di ulivo, ma anche di rosacea e di mirto ${ }^{146}$,

138 Chieti, 1997: 100.

139 Isernia, 1980: 216 nn. 70.4-5. Probabilmente riferibile ad armamento difensivo, ma forse non a un cinturone, è il gancio in bronzo edito in Isernia, 1980: $207 \mathrm{n}$. 68.2. Per un recente inquadramento del santuario: Capini, 2003.

140 Isernia, 1980: 208 n. 65.5 a-c.

141 Matteini Chiari, 2000: 285 allude genericamente alla presenza di "armi d'offesa" nel santuario. Stando a quanto edito in Sepino, 2004: 118 nn. 198-200, si tratta di un'ascia e di un giavellotto miniaturistici e di un Saurotèr.

142 Cassino, 2003: 24, 60, 64-65.

143 Milano, 1991: 164 nn. d43 a-c; Larino, 2003: 34-35 nn. A17-A19.

144 Considerazioni sulla determinazione della sfera funzionale di questi oggetti in Sannibale, 1998: 239 ss.

145 Da ultimo Tagliamonte, 2004: 105 ss., con rinvii alla la presenza di corone auree a foglie di ulivo è documentata anche nel non lontano santuario pentro di Schiavi d'Abruzzo ${ }^{147}$.

In anni più o meno recenti, diversi studiosi ${ }^{148}$ hanno evidenziato, soprattutto in rapporto all'ambiente magno-greco, la pluralità di significati e di usi, che tanto sul piano ideologico quanto su quello dei concreti comportamenti sociali, il riferimento alla corona può assumere (atletismo, guerra, simposio, contesti iniziatici, ecc.), fermo restando che essa rappresenta in primo luogo un elemento centrale della simbologia della vittoria, sia essa bellica o atletico/agonale ${ }^{149}$. Nel caso specifico del mondo sannitico, alla luce dei pochi dati sinora noti, sembra di potere dire che quella ampiezza di significati e di usi attestati in ambito italiota si sia venuta polarizzando proprio su questa dimensione bellica o atletico/agonale della vittoria. Se solo in anni recenti le sepolture sannitiche, in particolare quelle di area frentana ${ }^{150}$, hanno iniziato a restituire frammenti di corone, che, unitamente alla deposizione nei corredi di strigili e vasi per unguento, attestano la diffusione di nuovi modelli di autorappresentazione elitaria ${ }^{151}$ ispirati agli ideali dell'atletismo greco, le indicazioni provenienti dai santuari sembrerebbero invece, andare in altra direzione. In assenza di espliciti e specifici riferimenti alla paideia atletica nei contesti votivi, sembrerebbe infatti di potere supporre che il significato della presenza di corone preziose, offerte agli dei, quale segno di vittoria, vada prioritariamente definito, almeno per quanto riguarda gli esemplari più antichi ${ }^{152}$, in riferimento alla sfera bellica e/o agonale, ancorché non si possano del tutto escludere, specie nel caso degli esemplari di epoca più recente, altre possibili soluzioni ${ }^{153}$. A orientare in tal senso l'interpretazione, oltre alla

bibliografia precedente, alla quale aggiungi ora anche Larino, 2003: 26-36.

146 Milano, 1991: 163 nn. d42 a-e, 380 tav. XII.

147 Chieti, 1997: 117 nn. 1-3, tav. XII; Schiavi d'Abruzzo, 2001: 62 .

148 Ad es., Masiello, 1986; de La Genière, 1989; Blech, 1992; Coen, 1997; Guzzo, 2003.

149 Risultando queste ultime due sfere, peraltro, tra loro strettamente connesse.

150 Tagliamonte, 1997: 209; Larino, 2003: 20-26, in particolare 25 n. A7.7 (Larino, loc. Carpineto, tomba 19).

151 Dato che parrebbe segnalato anche dall'adozione del rituale dell'incinerazione in stàmnoi di bronzo: Larino, 2003: 20-23.

152 Entro il IV sec. a.C. sembrerebbero databili le due foglie di ulivo in argento da Campochiaro: Milano, 1991: 163 n. d42 a.

153 Che, ad es., chiamino in causa contesti di tipo inizia- 
pittura funeraria e vascolare campana e pestana nelle quali, come è noto, la corona compare di frequente come insegna di status, simbolo di vittoria militare ed elemento di eroizzazione del guerriero vincitore ${ }^{154}$, concorre (o sembrerebbe concorrere) la specifica valenza militare che, sul piano ideologico e simbolico, il riferimento alla corona assume in rapporto all'èthnos stesso dei Sanniti, almeno nella versione propagandistica e filellenica che ne viene proposta nell'immagine (punta di giavellotto in corona di alloro) posta sul verso degli oboli a legenda Saunitàn, databili ancora entro il terzo venticinquennio del IV sec. a.C. ${ }^{155}$.

A un sommario esame dei contesti santuariali sannitici che hanno restituito armi, sembrerebbe dunque di potere dire che, tanto per la eterogenea composizione dei complessi votivi quanto per tipologia e classi dei materiali attestati, non vi siano elementi tali da connotare specificamente in chiave militare o politico-militare quei contesti stessi. Quella bellica non è che una delle sfere di attività sociale in essi rappresentate, peraltro neppure quella prevalente. Nei materiali votivi di questi santuari è l'intero corpo sociale, l'universo devozionale maschile e femminile locale, con tutte le sue stratificazioni e articolazioni, a riflettersi e a rispecchiare la propria identità. In tale ambito, pertanto, la dedica di armi ${ }^{156}$, sempre anonima, più che alla consacrazione di spolia hostium, fa piuttosto pensare ad atti privati di devozione a valenza gratulatoria (a veri e propri ex voto, par transformation, secondo la definizione datane da J.-P. Morel) $)^{157}$, a offerte individuali di armi (simboliche e reali) proprie del dedicante. Il tema della vittoria bellica è comunque forse presente, se l'interpretazione proposta per le corone coglie nel segno. Nel caso di armi a esclusivo o forte valore simbolico, come quelle miniaturistiche e, almeno in una certa misura, gli stessi cinturoni $^{158}$, c'è poi da chiedersi se tali dediche non possano essere indizio di un regime dell'offerta di tipo diverso, legato piuttosto a situazioni che fanno riferimento alla strutturazione delle società locali per classi di età, ovvero che rinviano a contesti di iniziazione sociale o militare, a riti di passaggio, che dovevano o potevano trovare la loro sede nel santuario stesso.

Diversa è, come visto, la situazione di Pietrabbondante, i cui materiali votivi ci restituiscono certo un'immagine meno corale e articolata della comunità che in essi si rispecchiava. Qui l'attenzione è prevalentemente concentrata, per lo meno a partire da un certo momento, sulla dimensione collettiva e pubblica della dedica, ancorché quella privata non sia assente (ma comunque minoritaria). Per molti aspetti, come è stato fatto notare, Pietrabbondante rappresenta davvero "il luogo di culto pubblico", "il santuario del popolo in armi" ${ }^{159}$, venendo a svolgere in seno ai Sanniti Pentri quella preminente funzione politica e religiosa che, in passato, gli fece attribuire l'etichetta di santuario "federale"160. La guerra, l'evento bellico rappresentano l'ambito sul quale, per fattori strutturali e contingenze storiche, si polarizzano le attività cultuali e devozionali che in esso hanno sede. Il carattere specificamente militare e "trionfale" dell'ideologia che segna le manifestazioni che in esso si svolgono orienta il regime dell'offerta, incentrato sulla dedica di armi, siano esse skỳla o làphyra apò tòn polemion o piuttosto òpla ois autòs echreito, consacrate da singoli guerrieri. È attraverso tali dediche che principalmente trova espressione il tema della celebrazione della vittoria bellica. L'ideologia della vittoria, del resto, pervade così fortemente di sé il culto, da fare con ogni probabilità assimilare una delle divinità titolari del santuario a quella Afrodite Nikephòros ${ }^{161}$, la cui statua fu trasportata da Touxion (ovvero Cominium Tuticum, cioè Pietrabbondante) ${ }^{162}$ a Roma per opera di Q. Fabius Maximus Gurges ${ }^{163}$, all'epoca della terza guerra sannitica. Né mancano, come visto, fra i materiali votivi del santuario, in modo specifico fra le armi, espliciti riferimenti alla vittoria, alla Nike greca, simbolo come detto di successo bellico e atletico/agonale: alla più volte menzionata ala di elmo suditalico-calcidese con

Romito, 1995: 12-14; 2000: 192; Suano, 2000: 187-188.

159 La Regina, 1989: 422.

160 Lejeune, 1972.

161 Cfr. supra nt. 106.

162 Sull'identificazione di Pietrabbondante con Cominium (Tuticum), già proposta dall'antiquaria ottocentesca e in anni più recenti da $\mathrm{A}$. La Regina, vedi da ultimo Colonna, 1996: 128.

163 Secondo quanto è tramandato in un passo (37 b) dei Parallela minora pseudoplutarchei.

158 Sul valore simbolico del cinturone "sannitico" vedi in particolare le osservazioni di Suano, 1991: 138-139; 
figurazione di Nike alata si può accostare, ad esempio, un bel gancio di cinturone "sannitico" con corpo a figura umana riproducente una Nike alata $^{164}$. Ancora all'epoca del bellum sociale il richiamo alla vittoria sarà bene evidente, come attesta la dedica su lamina bronzea di un donario, nei pressi del tempio $\mathrm{B}$, alla Vittoria (Vikturrai) ${ }^{165}$ : quest'ultima appare ormai assimilata, nel nome e presumibilmente nelle prerogative, forse per un meccanismo di emulazione competitiva, alla Victoria romana, cui spettano, come ha mostrato T. Hölscher ${ }^{166}$, specifiche e ormai pressoché esclusive funzioni di garante e simbolo della vittoria bellica.

Proprio eventi come le guerre sannitiche e il bellum sociale sembrano avere costituito le occasioni e le circostanze nelle quali deve essersi andata fissando, nei suoi contenuti essenziali, quella specifica valenza politica e militare, che, unita al ruolo che nella mitistoria dei Sanniti sembrerebbe attribuito a Pietrabbondante, fece di questa il luogo dove si condensava la memoria storica e culturale di quel popolo ${ }^{167}$, o, per dirla con Pierre Nora ${ }^{168}$, un vero e proprio lieux de mémoire. Una memoria collettiva, un sentimento di identità, un senso di solidarietà tribale che, in momenti di gravi crisi e di minaccia esterna, proprio la vista delle armi sottratte ai nemici o dedicate dai propri padri doveva contribuire a mantenere vivi e rinsaldare.

\section{Abbreviazioni Bibliografiche}

Le abbreviazioni usate per i periodici sono quelle della Archäologische Bibliographie o si uniformano ad esse.

ADAM, A.-M. (1982): "Remarques sur une série de casques de bronze ou Tarente et les barbares dans la deuxième moitié du IV ${ }^{\mathrm{e}}$ s. av. J.-C.”. MEFRA 94, 732 .

ADAM, A.-M. (1986): "Emprunts et échanges de certains types d'armement entre l'Italie et le monde non méditerranéen aux $\mathrm{V}^{\mathrm{e}}$ et $\mathrm{IV}^{\mathrm{e}}$ siècles avant J.-C.”.

Adam, A.-M., Rouveret, A. (eds.): Guerre et sociétés en Italie $\left(V^{e}-I V^{e}\right.$ s. avant J.-C.), Actes de la Table ronde (Paris, 5 mai 1984), 19-28. Paris.

ADAM, A.-M. (2000): "Le bûcher de Patrocle et l'ostentation des armes dans les sociétés indigènes d'Italie méridionale". Ktema 25, 123-132.

164 Già edito in Fiorelli, 1869: 9 n. 135, il gancio è stato, fra l'altro, ripubblicato in Isernia, 1980: 152 n. 41.44.

165 Rix, 2002: 85 n. Sa 24, con bibliografia. Nel medesimo contesto storico, l'immagine della Vittoria viene, poi, riproposta nella monetazione dei ribelli italici:
Adamesteanu, D., Dilthey, H. (1992): Macchia di Rossano. Il santuario della Mefitis. Rapporto preliminare. Galatina.

Aitken, A. (1982): "A New Cheekpiece". AntK 25, 58-61.

Assmann, J. (1992): Das kulturelle Gedächtnis. Schrift, Erinnerung und politische Identität in frühen Hochkulturen. München; trad. it. La memoria culturale. Scrittura, ricordo e identità politica nelle grandi civiltà antiche, Torino 1997.

Baitinger, H. (2001): Die Angriffswaffen aus Olympia (Olympische Forschungen, 29). Berlin-New York.

BArringer, J.M. (1995): Divine Escorts. Nereids in Archaic and Classical Greek Art. Ann Arbor.

Benassai, R. (2001): La pittura dei Campani e dei Sanniti. Roma.

BerLin (1988): Antike Helme. Sammlung Lipperheide und andere Bestände des Antikenmuseums Berlin. Mainz.

Berlingó, I., Russo Tagliente, A. (1992-1993): "Chiaromonte (Potenza). La necropoli arcaica in località Sotto La Croce, scavi 1973”. NSc 1992-1993, 233-407.

BESQUes, S. (1988): "Deux reliefs apuliens en terre cuite". MonPiot 69, 1-28.

Blech, M. (1992): Studien zum Kranz bei den Griechen. Berlin-New York.

Bordenache Battaglia, G. (1979): Le ciste prenestine, I.1. Roma.

Bordenache Battaglia, G., Emiliozzi, A. (1990): Le ciste prenestine, I.2. Roma.

Bottini, A. (1991): "Elmi 'suditalici-calcidesi'- Elmi 'a bottone”. Bottini, Fresa (1991), 97-99.

Bottini, A. (1992): "Metallotecnica". AA.VV.: Introduzione all'artigianato della Puglia antica dall'età coloniale all'età romana, 139-160. Bari.

Bottini, A. (1999): "Una spada da Banzi”. Ostraka 8, 920.

Bottini, A., Fresa, M.P. (eds.) (1991): Forentum II. L'acropoli in età classica. Venosa.

Bottini, A., Rainini, I., Isnenghi Colazzo, S. (1976): "Rocca S. Felice (Avellino). - Il deposito votivo del santuario di Mefite". NSc 1976, 359-524.

Campana, 1987: 90 ss.

166 Hölscher, 1967.

167 Assmann, 1992 (1997).

168 Nora, 1984. 
CAHn, D. (ed.) (1989): Waffen und Zaumzeug, catalogo della mostra. Basel.

CAhn, D. (1990): VAN Bothmer, D. (ed.): Glories of the Past. Ancient Art from the Shelby and Leon Levy Collection, 114-122 n. 95. New York.

Campana, A. (1987): La monetazione degli insorti italici durante la guerra sociale (91-87 a.C.). Modena.

CAPINI, S. (1991): "Il santuario di Pietrabbondante". MILANO (1991), 113-114.

CAPINI, S. (2003): "Il santuario di Ercole a Campochiaro". Santuari e luoghi di culto nell'Italia antica, 233-250. Roma.

Capini S., DE Benedittis, G. (2000): Pietrabbondante. Guida agli scavi archeologici. Campobasso.

CARABA, A. (1873): "Relazione sugli scavi di antichità in Pietrabbondante nel 1872". GiornScaviPompei n.s. 2.19, cc. 395-402.

Caraba, A., Cremonese, F.S. (1871): "Gli antichi monumenti di Pietrabbondante nel Molise. Relazione 1871”. Roma [1990].

Cardosa, M. (2002): "Il dono di armi nei santuari delle divinità femminili in Magna Grecia”. GiUMLIAMAIR, A., Rubinich, M. (eds.): Le arti di Efesto. Capolavori in metallo dalla Magna Grecia, 99-102. Milano.

Cardosa, M. (c. s.): "L'offerta di armi nei santuari di Kore-Persefone di area locrese". ROMA (c. s.).

CASSINO (2003): La via dei metalli, dalla materia alla forma tra il Melfa e il Rapido, catalogo della mostra. Roma.

Celano (2003): Campanelli, A. (ed.): La Collezione Torlonia di Antichità del Fucino, catalogo della mostra, Pescara.

Cerchiai, C. (1984): "Alcune osservazioni su due bronzi prenestini”. BdA 69, 53-58.

Chieti (1997): Campanelli, A., Faustoferri, A. (eds.): I luoghi degli dei. Sacro e natura nell'Abruzzo italico, catalogo della mostra. Pescara.

Cianfarani, V., Franchi Dell'Orto, L., La Regina, A. (1978): Culture adriatiche antiche di Abruzzo e Molise. Roma.

COARELli, F. (1976): "Un elmo con iscrizione latina arcaica al Museo di Cremona". L'Italie préromaine et la Rome républicaine. Mélanges offerts à Jacques Heurgon, I, 157-179. Rome.

Coen, A. (1997): "Elmi di bronzo e corone d'oro: una rara associazione simbolica nelle sepolture etrusche di IV secolo a.C.”. Miscellanea etrusco-italica II, 89107. Roma.

Colonna, G. (1996): "Alla ricerca della 'metropoli' dei
Sanniti”. Identità e civiltà dei Sabini, Atti del XVIII convegno nazionale di studi etruschi e italici (RietiMagliano Sabina 30 maggio-3 giugno 1993), 107130. Firenze.

D’Agostino, B. (1980): "Le armi. Le paragnatidi del nuovo scavo”. ISERNIA (1980), 140-142, 145-150.

DE FilipPIS, A. (1995): "Ceramica a decorazione policroma da Cuma”. Studi sulla Campania preromana, 81-97. Roma.

De La Genière, J. (1989): "Épire et Basilicate. À propos de la couronne d'Armento". MEFRA 101, 691-698.

De Petra, G. (1870): “Gli scavi di antichità in Pietrabbondante”. GiornScaviPompei n.s. 2.14, cc. 117-137.

D'Ercole, V., Copersino, M.R. (eds.) (2003): La necropoli di Fossa, IV. L'età ellenistico-romana. Pescara.

DinTis, P. (1986): Hellenistische Helme. Roma.

Di ViTA, A. (2003): "Ancora Casmene: una nota”. PP 58, 66-70.

ERICE (c. s.): Guerra e pace in Sicilia e nel Mediterraneo antico (VIII-III sec. a.C.): arti, prassi e teoria della pace e della guerra, Atti delle Quinte Giornate internazionali di studi sull'area elima e la Sicilia occidentale nel contesto mediterraneo (Erice, 12-15 ottobre 2003).

Feugère, M. (1993): Les armes des Romains de la République à l'Antiquité tardive. Paris.

FEugÈRe, M. (1994): "L'équipement militaire d'époque républicaine en Gaule”. JRMES 5, 3-23.

Fiorelli, G. (1869): Cataloghi del Museo Nazionale di Napoli. Armi antiche. Napoli.

GARruCCI, R. (1860): Bullinst, 8-9.

Giampaola, D. (1980): "Le armi. Gli elmi del Museo Nazionale di Napoli”. ISERNIA (1980), 139-140, 143-145.

Greco, E. (1970): Il Pittore di Afrodite. Roma.

GRECO, E. (1991): "In Lucania: ruoli dei sessi e istituzioni politico-religiose (a proposito del Santuario di Torre Satriano)". DArch s. III, 9, 75-83.

Greco, E., Guzzo, P.G. (eds.) (1992): Laos II. La tomba a camera di Marcellina. Napoli-Taranto.

GuZzI, O. (2002): "Note su alcune composizioni narrative nella ceramica volsiniese". Prospettiva, 106-107, 118-125.

Guzzo, P.G. (1990): "L'elmo da Pacciano. Ipotesi sulla circolazione delle armi decorate ellenistiche". BdArch 2, 1-13.

Guzzo, P.G. (1993): "L'armamento in Lucania fra IV e 
III secolo”. MELFI (1993), 159-171.

Guzzo, P.G. (2001): “Armi e società”. ROMA (2001), 2327.

Guzzo, P.G. (2003): "Corone d'agone, tra guerra e morte, in Magna Grecia”. A. LA REGINA (ed.): Nike. Il gioco e la Vittoria, catalogo della mostra, 92103. Milano.

HÖlSCHER, T. (1967): Victoria Romana. Mainz am Rhein.

Hölscher, T. (2003): "Images of War in Greece and Rome: Between Military Practice, Public Memory, and Cultural Symbolism”. JRS 93, 1-17.

Isernia (1980): Sannio. Pentri e Frentani dal VI al I sec. a.C., catalogo della mostra. Roma.

Isernia (1984): Sannio. Pentri e Frentani dal VI al I sec. a.C., Atti del convegno (Isernia, 10-11 novembre 1980). Campobasso.

JaCKSON, A.H. (1991): "Hoplites and the Gods: The Dedication of Captured Arms and Armour". HANSON V.D. (ed.): Hoplites. The Classical Greek Battle Experience, 228-249. London-New York.

JaCQUemin, A. (1999): "Guerres et offrandes dans les sanctuaires". Pallas 51, 141-157.

JaCQUemin, A. (2000): Guerre et religion dans le monde grec: 490-322 av. J.-C. Paris.

Johannowsky, W. (2004): "Materiale di età arcaica e classica da Rufrae, S. Agata dei Goti, Circello, Casalbore, Carife, Castel Baronia, Bisaccia, Morra De Santis". CAIAZZA, D. (ed.): Safinim. Studi in onore di Adriano La Regina per il premio I Sanniti, 275311. Piedimonte Matese (CE).

Jost, M. (1999 a): "Les divinités de la guerre". PROST, F. (ed.): Armées et sociétés de la Grèce classique. Aspects sociaux et politiques de la guerre aux $V^{e}$ et $I V^{e}$ s. av. J.C., 163-178. Paris.

Jost, M. (1999 b): “Guerre et religion". Pallas 51, 129139.

Junkelmann, M. (2000): Römische Helme. Berlin-Mainz am Rhein.

Kunze, E. (1967): "Chalkidische Helme”. VIII Bericht über die Ausgrabungen in Olympia, Berlin, 133-183.

KunZE, E. (1991): Beinschienen (Olympische Forschungen, 21). Berlin-New York.

KunZE, E. (1994): "Chalkidische Helme IV-VII, mit Nachträgen zu I und II". IX Bericht über die Ausgrabungen in Olympia, 27-100. Berlin-New York.

KunZL, E. (1997): "Waffendekor in Hellenismus". JRMES 8, 61-89.

LA Regina, A. (1976): "Il Sannio". Zanker, P. (ed.):
Hellenismus in Mittelitalien, Kolloquium (Göttingen, vom 5. bis 9. Juni 1974), 219-244. Göttingen.

LA Regina, A. (1980): "Pietrabbondante". Isernia (1980), 131.

La Regina, A. (1984): "Aspetti istituzionali nel mondo sannitico". ISERNIA (1984), 17-25.

LA Regina, A. (1989): "I Sanniti". Italia omnium terrarum parens, 301-432. Milano.

LA Regina, A. (1990): "Safinim. Dal conflitto con Roma alla tota Italia". PAONE, N. (ed.): Il Molise. Arte, cultura, paesaggi, 31-53. Roma.

LARINO (2003): Lo sport nell'Italia antica. Dai Sanniti ai Longobardi, catalogo della mostra. Ripalimosani (CB).

LA TORRe, G.F. (2002): Un tempio arcaico nel territorio dell'antica Temesa. L'edificio sacro in località Imbelli di Campora San Giovanni. Roma.

LA TORRE, G.F. (c. s.): "Le lance di Temesa e le offerte di armi nei santuari magno-greci e sicelioti in età arcaica”. ROMA c. s.

LE BoHec, Y. (1997): "L'armement des Romains pendant les Guerres Puniques”. JRMES 8, 13-24.

Lentini, M.C. (2000): "Armi a Naxos dalle mura e dal santuario". Damarato. Studi di antichità classica offerti a Paola Pelagatti, 155-166. Milano.

Lejeune, M. (1972): "Études et mémoires. I. Notes de linguistique italique. XXXI. Sur l'aspect fédéral du sanctuaire de Calcatello". REL 50, 94-111.

LiPPOLIS, E. (1984): “La toreutica”. MILANO (1984), 3150.

LOMBARDO, M. (c. s.): "Les armes dans les sanctuaires du monde grec: quelques aspects". Les armes dans l'Antiquité: de la technique à l'imaginaire, Actes du Colloque internationale (Montpellier, 20-22 mars 2003).

LONIS, R. (1979): Guerre et religion en Grèce a l'époque classique. Paris.

MahÉ, M. (1999): "Le pythagorisme d'Italie du Sud vue par Tite-Live”. Ktema 24, 147-157.

MAIURI, A. (1913): "Scavi nell'area di un tempio italico nell'antica Bovianum Vetus". NSc, 456.

MANCINI, C. (1899): "Il linguaggio simbolico della regina delle epigrafi osche scoverto ed interpretato". AttiRealAcc Arch Lett BellArtNapoli 20.3, 1-44.

MAnNino, K. (c. s.): "L'iconografia del guerriero nel mondo apulo". TARANTO (2004), 699-726.

Martelli, M. (2003): "Armi miniaturistiche da Ialysos". Fiorentini, G., Caltabiano, M., Calderone, A 
(eds.): Archeologia del Mediterraneo. Studi in onore di Ernesto De Miro, 467-472.

Masiello, L. (1984): "Le corone”. MILANO (1984), 71108.

Masseria, C. (1999): “... et Venerem et proelia destinat... (Hor. carm. III, 13, 5). Riti di passaggio in un santuario di Banzi”. Ostraka 8, 468-490.

Matteini Chiari, M. (2000): "Il santuario italico di San Pietro di Cantoni di Sepino“. SANNTini (2000), 280291. Roma.

MAZZEI, M. (1996): “Le armi”. TARANTO (1996), 119128.

Melfi (1993): Bottini, A. (ed.): Armi. Gli strumenti della guerra in Lucania, catalogo della mostra. Bari.

Mele, A. (2000): "Archita e Gaio Ponzio sannita". Tortorelli Ghidini, M., Storchi Marino, A., ViscontI, A.: Tra Orfeo e Pitagora. Origini e incontri di culture nell'antichità, Atti dei seminari napoletani 1996-1998, 433-444. Napoli.

Mele, A. (2002): "Taranto dal IV secolo a.C. alla conquista romana”. TARANTO (2002), 79-99.

MichetTI, L.M. (2003): Le ceramiche argentate e a rilievo in Etruria nella prima età ellenistica (MonAnt s. misc. VIII, 61). Roma.

Milano (1984): De Juliıs, E. (ed.): Gli ori di Taranto in età ellenistica, catalogo della mostra. Milano.

Milano (1991): CaPini, S., Di Niro, A. (eds.), Samnium. Archeologia del Molise, catalogo della mostra. Roma.

Minervini, G. (1858): "Notizie delle nuove scavazioni in Pietrabbondante”. BArchNap n.s. 6. 24, 185-190.

Morel, J.-P. (1992): "Ex-voto par transformation, ex voto par destination (à propos du dépôt votif de Fondo Ruozzo à Teano)". Mactoux, M.M., GenY, E. (eds.): Mélanges Pierre Lévêque, VI, 221-232. Besançon.

Morel, J.-P. (2002): "Taranto nel Mediterraneo in epoca ellenistica”. TARANTO (2002), 529-574.

MoustakA, A. (1994): "Ein votivschild aus dem Heraion von Samos. Zum Verhältnis zwischen Vasenmalerei und Toreutik in Ionien”. AM 109, 11-37.

NAPOLI (1996): I Greci in Occidente. La Magna Grecia nelle collezioni del Museo Archeologico di Napoli, catalogo della mostra. Napoli.

Nava, M.L., Osanna, M. (eds.) (2001): Rituali per una dea lucana. Il santuario di Torre Satriano. Potenza.

Nava, M.L., Poccetti, P. (2001): "Il santuario lucano di Rossano di Vaglio: una nuova dedica osca". MEFRA 113, 95-122.
NorA, P. (1984): "Entre mémoire et histoire". ID. (ed.), Les lieux de la mémoire, I, XVII-XLII. Paris.

OsAnNA, M. (2001): "Guerra e religione tra mondo greco e mondo indigeno”. ROMA (2001), 63-67.

PANCIERA, S. (1998): "Epigrafia. Una voce soppressa". ArchCl 50, 313-330.

PAPI, R. (2000): "Continuità e trasformazione dell'ideologia militare nei territori sabellici medioadriatici". SANNTINI (2000), 138-165.

Parra, M.C. (1996): "I culti di Hipponion". RegGio Calabria (1996), 139-141.

PARRA, M.C. (c. s.): “Armi per Una dea in Magna Grecia: alcune considerazioni a proposito di nuove testimonianza kauloniati”. ERICE c. s.

Pflug, H. (1988 a): "Chalkidische Helme". BerLin (1988), 137-150

Pflug, H. (1988 b): "Italische Helme mit Stirnkehle". BERLIN (1988), 276-292.

Pflug, H. (ed.) (1989): Schutz und Zier. Helme aus dem antikenmuseum Berlin und Waffen anderer Sammlungen, catalogo della mostra, Basel.

Pfrommer, M. (1982): "Grossgriechiscen und Mittelitalischer Einfluss in der Rankenornamentik frühhellenistischer Zeit”. JdI 97, 119-190.

Philipe, H. (1994): "Olympia, Die Peloponnes und die Westgriechen”. JdI 109, 77-92.

Philipp, H. (2004): Archaische Silhouettenbleche und Schildzeichen in Olympia (Olympische Forschungen, 30). Berlin-New York.

PoccetTI, P. (2001): “Armi e guerra tra le popolazioni indigene della Magna Grecia". ROMA (2001), 4955 .

Polito, E. (1998): Fulgentibus armis. Introduzione allo studio dei fregi d'armi antichi. Roma.

Pontrandolfo, A., Rouveret, A. (1992): Le tombe dipinte di Paestum, Modena.

Pritchett, W.K. (1979): The Greek State at War, III. Berkeley-Los Angeles-London.

Quesada SAnZ, F. (1997): "Montefortino-type and Related Helmets in the Iberian Peninsula: A Study in Archaeological Context”. JRMES 8, 151-166.

Reggio Calabria (1996): Lattanzi, E., ET AL. (eds.), I Greci in Occidente. Santuari della Magna Grecia in Calabria. Napoli.

RIx, H. (2002): Sabellische Texte. Heidelberg.

Roma (1973): Roma medio repubblicana, catalogo della mostra. Roma 
Roma (1981): Enea nel Lazio. Archeologia e mito, catalogo della mostra. Roma.

Roma (2000): Italia dei Sanniti, guida alla mostra. Roma.

Roma (2001 a): LA Regina, A. (ed.): Sangue e arena, catalogo della mostra. Roma.

Roma (2001 b): Genti in arme. Aristocrazie guerriere della Basilicata antica, catalogo della mostra. Roma.

Roma (c. s.): Les rites de victoire (IVe siècle avantJ.-C.-Ier siècle après J.-C.), Actes du Colloque (Rome, 19, 20, 21 avril 2001).

Romito, M. (1995): I cinturoni sannitici. Napoli.

Romito, M. (2000): "I cinturoni sannitici". SANNiti (2000), 192-201.

Rouveret, A. (2000): “Captiva arma: guerre, butin, économie dans les cités de Grande Grèce et de Campanie du Ve siècle à l'expédition de Pyrrhus". Andreau, J., Briant, P., Descat, R. (eds.): Économie antique. La guerre dans les économies antiques, 83-102. Saint-Bertrand-de-Comminges.

Ruggiero, M. (1888): Degli scavi di antichità nelle province di terraferma dell'Antico Regno di Napoli dal 1743 al 1876. Napoli.

Russo, A. (1988): "Armi e strumenti". GIORGI, M. ET AL.: Forentum I. Le necropoli di Lavello, 247-253. Venosa.

Russo, A. (1995): "Armento. Archeologia di un centro indigeno". BdArch 35-36.

Russo, A. (2001): "L'arte della guerra tra IV e III secolo a.C.”. ROMA (2001), 57-61.

Sabbione, C. (1996 a): "Il santuario di Persefone in contrada Mannella”. Reggio CAlabria (1996), 32-34.

Sabbione, C. (1996 b): "Medma, loc. Greci, stipe votiva località Calderazzo”. REGGIO CALABRIA (1996), 110111.

SAbBione, C. (1996 c): "Hipponion: il deposito votivo in località Scrimbia”. RegGiO CALABRIa (1996), 155161.

SAnnibale, M. (1995): "Cinturoni italici della collezione Gorga”. MEFRA 107, 937-1020.

Sannibale, M. (1998): Le armi della Collezione Gorga al Museo Nazionale Romano. Roma.

SANNTINI (2000): Studi sull'Italia dei Sanniti. Roma.

SAUlNier, Chr. (1983): L'armée et la guerre chez les peuples samnites $\left(V I I^{e}-I V^{e}\right.$ si.). Paris.

Schiavi D'Abruzzo (2001): Schiavi d'Abruzzo. "Verso la cima del monte". I templi italici. L'ambiente e il territorio. L'archeologia e la storia. San Giovanni Teatino
$(\mathrm{CH})$.

Schneider Hermann, G. (1996): Herring, E. (ed.), The Samnites of the Fourth Century B.C. as depicted on Campanian Vases and in Other Sources. London.

Sepino (2004): Matteini Chiari, M. (ed.): La Dea, il Santo, una Terra. Materiali dallo scavo di San Pietro di Cantoni di Sepino. Roma.

Spatafora, F. (2000): "Indigeni, Punici e Greci in età arcaica e tardo-arcaica sulla Montagnola di Marineo e nella valle dell'Eleuterio". Atti delle terze giornate internazionali di studi sull'area elima (Gibellina-EriceContessa Entellina, 23-26 ottobre 1997), 895-918. Pisa-Gibellina.

Spatafora, F. (c. s.): "Vincitori e vinti: sulle deposizioni di armature nella Sicilia di età arcaica”. ERICE c. s.

Storti, S. (1994): "Pietrabbondante". Nenci, G., VAllet, G. (eds.), Bibliografia topografica della colonizzazione greca in Italia e nelle isole tirreniche, 13, 553562. Pisa-Roma.

Strazzulla, M.J. (1973): Il santuario sannitico di Pietrabbondante. Roma.

SuAno, M. (1986): Sabellian-Samnite Bronze Belts in the British Museum. London.

SuANO, M. (1991): "Alcune osservazioni sui cinturoni in bronzo di tipo sannitico”. MILANO (1991), 135139.

SuAno, M. (2000): "Il cinturone sabellico-sannita come abbigliamento sociale”. SANNITI (2000), 183-191.

Tagliamonte, G. (1989-1990): "Iscrizioni votive italiche su armi”. SciAnt 3-4, 519-534.

Tagliamonte, G. (1994 a): I figli di Marte. Mobilità, mercenari e mercenariato italici in Magna Grecia e Sicilia. Roma.

TAgliamonte, G. (1994 b): "Sinistrum crus ocrea tectum”. StEtr 60, 125-141.

Tagliamonte, G. (1997): I Sanniti. Caudini, Irpini, Pentri, Carricini, Frentani. Milano.

Tagliamonte, G. (1999): "Lo sviluppo di una società aristocratica: il ruolo delle armi”. Piceni. Popolo d'Europa, catalogo della mostra, 112-114. Roma.

Tagliamonte, G. (2003 a): "La terribile bellezza del guerriero". I Piceni e l'area medio-adriatica, Atti del XXII Convegno di studi etruschi ed italici (Ascoli Piceno-Teramo-Celano-Ancona, 9-13 aprile 2000), 533-553. Pisa-Roma.

TAgliamonte, G. (2003 b): "Note sulla circolazione degli elmi nell'Abruzzo e nel Molise preromani”. MEFRA 115, 129-175. 
Tagliamonte, G. (2004): "Horsemen and Dioskouroi Worship in Samnite Sanctuaries”. JONES, H. (ed.): Samnium. Settlement and Cultural Change, 103-114. Providence (Rh. I.).

TARAnto (1996): Lippolis. E. (ed.): I Greci in Occidente. Arte e artigianato in Magna Grecia, catalogo della mostra. Napoli.

TARAnto (2002): Taranto e il Mediterraneo, Atti del XLI Convegno di studi sulla Magna Grecia (Taranto, 1216 ottobre 2001). Taranto-Napoli.

TARAnto (2004): Alessandro il Molosso e $i$ "condottieri” in Magna Grecia, Atti del XLIII Convegno di studi sulla Magna Grecia (Taranto-Cosenza, 26-30 settembre 2003). Taranto-Napoli.

TARDITI, C. (1996): "Il vasellame". TARANTO (1996), 105115.

VAn Wonterghem, F. (1984): Superaequum, Corfinium,
Sulmo (Forma Italiae, IV.1). Firenze.

Venezia (1996): Pugliese Carratelli, G. (ed.): I Greci in Occidente, catalogo della mostra.

Vermeule, C.C., ET AL. (1988): Sculpture in Stone and Bronze in the Museum of Fine Arts, Boston. Additions to the Collections of Greek, Etruscan, and Roman Art 19711988. Boston.

Vitali, D. (1992): Tombe e necropoli galliche di Bologna e territorio. Bologna.

Voкотороulou, J. (1982): "Phrygische Helme". AA 93, 497-520.

WAURICK, G. (1988): "Helme der hellenistischer Zeit und ihre Vorläufer”. BERLIN (1988), 163-180.

Zhmodikov, A. (2000): "Roman Republican Infantrymen in Battle (IV-II Centuries BC)". Historia 49, 67-78.

\section{Addendum}

Nelle more di stampa sono venuto a conoscenza dell'esistenza dell'articolo di A.-M. Adam, "Dépôts d'armes dans les sanctuaires italiques de $\mathrm{IV}^{\mathrm{e}}$ au $\mathrm{I}^{\mathrm{er}}$ siècle avant J.-C.”, di cui tuttavia non ho avuto modo di prendere visione. 